\title{
Improved Plant Nitrate Status Involves in Flowering Induction by Extended Photoperiod
}

\author{
Jia Yuan Ye ${ }^{1}$, Wen Hao Tian', Miao Zhou ${ }^{1}$, Qing Yang Zhu ${ }^{1}$, Wen Xin Du' and \\ Chong Wei Jin ${ }^{1 *}$
}

${ }^{1}$ State Key Laboratory of Plant Physiology and Biochemistry, College of Natural Resources and Environmental Science, Zhejiang University, Hangzhou, China, ${ }^{2}$ State Key Laboratory of Plant Physiology and Biochemistry, College of Life Science, Zhejiang University, Hangzhou, China

\section{OPEN ACCESS}

Edited by: Hong-Qing Ling,

Institute of Genetics

and Developmental Biology, Chinese Academy of Sciences, China

Reviewed by:

Xiaorong Fan,

Nanjing Agricultural University, China

Francesco Mercati,

Institute of Biosciences

and Bioresources, National Research

Council (CNR), Italy

${ }^{*}$ Correspondence:

Chong Wei Jin

jincw@zju.edu.cn

Specialty section:

This article was submitted to

Plant Nutrition,

a section of the journal

Frontiers in Plant Science

Received: 16 November 2020

Accepted: 19 January 2021

Published: 12 February 2021

Citation:

Ye JY, Tian WH, Zhou M, Zhu QY,

Du WX and Jin CW (2021) Improved

Plant Nitrate Status Involves

in Flowering Induction by Extended

Photoperiod.

Front. Plant Sci. 12:629857.

doi: 10.3389/fpls.2021.629857
The floral transition stage is pivotal for sustaining plant populations and is affected by several environmental factors, including photoperiod. However, the mechanisms underlying photoperiodic flowering responses are not fully understood. Herein, we have shown that exposure to an extended photoperiod effectively induced early flowering in Arabidopsis plants, at a range of different nitrate concentrations. However, these photoperiodic flowering responses were attenuated when the nitrate levels were suboptimal for flowering. An extended photoperiod also improved the root nitrate uptake of by NITRATE TRANSPORTER 1.1 (NRT1.1) and NITRATE TRANSPORTER 2.1 (NRT2.1), whereas the loss of function of NRT1.1/NRT2.1 in the nrt1.1-1/2.1 2 mutants suppressed the expression of the key flowering genes CONSTANS (CO) and FLOWERING LOCUS T $(F T)$, and reduced the sensitivity of the photoperiodic flowering responses to elevated levels of nitrate. These results suggest that the upregulation of root nitrate uptake during extended photoperiods, contributed to the observed early flowering. The results also showed that the sensitivity of photoperiodic flowering responses to elevated levels of nitrate, were also reduced by either the replacement of nitrate with its assimilation intermediate product, ammonium, or by the dysfunction of the nitrate assimilation pathway. This indicates that nitrate serves as both a nutrient source for plant growth and as a signaling molecule for floral induction during extended photoperiods.

Keywords: nitrogen, photoperiodic flowering responses, suboptimal nitrate supply, NRT1.1, NRT2.1, FLOWERING LOCUS T, CONSTANS, nitrate uptake

\section{INTRODUCTION}

The transition from vegetative to reproductive development is a pivotal event in the lives of annual plants and can have a profound impact on their fertility and population sustainability (Guo and Yang, 1998; Lin and Tsay, 2017). Floral induction is regulated by both environmental and endogenous cues. The isolation of loss-of-function mutations and the analysis of transgenic plants, has resulted in hundreds of genes that are related to the timing of flowering being classified into several distinct pathways in Arabidopsis thaliana, including photoperiod, vernalization, gibberellin acid, autonomous, age, sugar, and temperature pathways (Fornara et al., 2010; 
Srikanth and Schmid, 2011; Kim et al., 2012; Wahl et al., 2013; Capovilla et al., 2014; Yuan et al., 2016; Luo et al., 2018; Andrés et al., 2020; Bao et al., 2020). These pathways were found to converge on the "integrator" genes SUPPRESSOR OF OVEREXPRESSION OF CO 1 (SOC1) and FLOWERING LOCUS T (FT) (Putterill et al., 1995; Luo et al., 2018; Andrés et al., 2020). Among the environmental cues, photoperiod, which integrates inputs from the circadian clock and light receptors, is the most important seasonal cue in floral induction (Bao et al., 2019). In most cases, angiosperms flower earlier in extended photoperiod conditions (Andrés and Coupland, 2012; Luo et al., 2018; Bao et al., 2019). Several studies have indicated that the flowering of Arabidopsis induced by the long-day (LD) treatments is under the control of the canonical genetic photoperiod pathway (Samach et al., 2000; Suárez-López et al., 2001; Fornara et al., 2010; Andrés and Coupland, 2012). Briefly, the FLAVIN-BINDING KELCH REPEAT F-BOX 1 (FKF1) perceives light signals and interacts with GIGANTEA (GI) to mediate the degradation of CONSTANS (CO) transcriptional repressors CYCLING DOF FACTORs (CDFs), which promote CO transcripts (Sawa et al., 2007; Fornara et al., 2009, 2010). The CO protein then interacts with the $\mathrm{B}$ and $\mathrm{C}$ subunits of Nuclear Factor Y (NF-Y) to form the NF-CO complex, which increases FT expression and thus initiates flowering (Wenkel et al., 2006; Tiwari et al., 2010; Andrés and Coupland, 2012; Gnesutta et al., 2017; Luo et al., 2018; Bao et al., 2019). Floral repressors are also involved in the regulation of photoperiodic flowering responses. For example, the AP2-like transcription factor SCHLAFMUTZE (SMZ) and its paralog, SCHNARCHZAPFEN (SNZ) delay flowering under LD conditions. Furthermore, chromatin immunoprecipitation experiments have shown that SMZ can directly bind to the FT locus, which leads to the downregulation of FT expression (Mathieu et al., 2009; Golembeski and Imaizumi, 2015).

In addition to the photoperiod, the availability of nitrogen $(\mathrm{N})$, which is an integral mineral element for plants and required in quantities higher than any other, is an important environmental factor that affects flowering time (Martínez et al., 2004; Achard et al., 2006; Castro Marín et al., 2011; Kant et al., 2011; Liu et al., 2013; Yuan et al., 2016; Zeng et al., 2017; Gras et al., 2018; Olas et al., 2019). The effects of nitrate on flowering have been intensively studied in recent decades. By summarizing previous studies and further examining the flowering time of Arabidopsis plants with various additional levels of nitrate, Lin and Tsay (2017) propose a U-shaped response of flowering to nitrate. They identified that an optimal level of nitrate facilitates flowering and that nitrate levels above or below this delayed flowering. Although further studies may be required to clarify whether the U-shaped response of flowering to nitrate can be generalized for natural conditions, it could be reasonably suspected that the factors affecting root nitrate acquisition may also influence flowering. Several studies have indicated that carbohydrate photosynthates, such as sucrose and trehalose-6phosphate (T6P), promote flowering (Ohto et al., 2001; Gómez et al., 2010; Moghaddam and van den Ende, 2013; Wahl et al., 2013; Andrés et al., 2020). Very recently, Olas et al. (2019) found that the knockdown of TREHALOSE PHOSPHATE SYNTHASE 1, which encodes the enzyme producing T6P, was unable to flower under limited $\mathrm{N}$ soil conditions but could flower under optimal $\mathrm{N}$ soil and short-day (SD) conditions, highlighting the combined importance of T6P and nitrate for floral induction. Interestingly, light conditions intensively affect nitrate acquisition by the roots. For example, nitrate uptake by soybean and barley plants could be increased by $150 \%$ and $300 \%$, respectively, under light conditions compared to dark conditions (Delhon et al., 1995; Peuke and Jeschke, 1998; Lejay et al., 1999). Furthermore, it was shown that the loss of function of a light-responsive bZIP transcription factor ELONGATED HYPOCPTYL5 (HY5) in Arabidopsis hy5 mutants, suppressed nitrate uptake by decreasing the level of carbon photo-assimilate sucrose (Chen et al., 2016). In contrast, the exogenous application of sucrose prevented the inhibition of NRT1.1 and NRT2.1 expression under dark conditions, thus significantly increasing nitrate uptake in the dark (Lejay et al., 1999, 2003). Theoretically, changes in the photoperiod could be expected to alter the production of carbon photo-assimilates in plants and may subsequently modify the nitrate uptake by roots. In this context, the mechanisms underlying the photoperiodic flowering responses are probably associated with alterations in the uptake of nitrate.

In well-aerated soils, nitrate is the primary $\mathrm{N}$ source, owing to rapid nitrification (Miller and Cramer, 2005), and the concentration of nitrate varies greatly in different soil environments, ranging from 0 to $1.8 \mathrm{mM}$, and even above $70 \mathrm{mM}$ (Crawford and Glass, 1998). However, in most natural habitats, such as forest and grassland ecosystems, soil nitrate concentrations are $<1 \mathrm{mM}$. In agricultural ecosystems, nitrate concentrations in soils are typically $1-5 \mathrm{mM}$, but insufficient $\mathrm{N}$ levels are common due to the rapid depletion of nitrate from soil solutions (Crawford and Glass, 1998; Miller and Cramer, 2005). Therefore, it is important to clarify if nitrate uptake plays a role in regulating photoperiod-modulated flowering when nitrate supplies are below the optimal levels, to more reasonably evaluate the impact of nitrate on the plant ecosystem and crop production. In this study, we investigated the above notion and demonstrated that the nitrate status of the soil is involved in photoperiod-modulated flowering when it is below the optimal level. Specifically, extended photoperiod conditions improved root nitrate uptake by NRT1.1 and NRT2.1, which subsequently upregulated the expression of the key light-responsive regulators, FT and $C O$, in plants, thereby contributing to premature flowering induction.

\section{EXPERIMENTAL PROCEDURES}

\section{Plant Material}

The Arabidopsis mutants nrt1.1-1 (Salk _097431), nrt2.1-2 (CS859604), and nia2-5 (CS2355) were obtained from the Arabidopsis Biological Resource Center. The $f t-10$ and co-1 mutants were kindly provided by Y.R. Hu (Xishuangbanna Tropical Botanical Garden, Chinese Academy of Sciences, China), and the cry1 mutant (CS6955) were kindly provided by S. Yuan (Sichuan Agricultural University, China) and B. Liu (Chinese Academy of Agricultural Sciences, Beijing, China). All mutants were all in Columbian (Col-0) background. The 
nrt1.1-1/2.1-2 mutants were generated by crossing nrt1.1-1 with nrt2.1-2, as described previously (Ye et al., 2019). As nitrate storage in seeds may affect their germination time, we prolonged the vernalization period to minimize the differences in the germination times between the different plant lines. Consequently, all the seeds used in this study were harvested at the same time and stored for approximately 1 year at $4^{\circ} \mathrm{C}$; furthermore, another 3 days of stratification were also conducted at $4^{\circ} \mathrm{C}$ before sowing the seeds.

\section{Plant Culture and Treatment Conditions}

For agar culturing, the surface-sterilized Arabidopsis seeds were sown on basal agar medium containing $1 \%(\mathrm{w} / \mathrm{v})$ sucrose, $0.8 \%$ $\operatorname{agar}(w / v)$ (Sigma), $\mathrm{NaH}_{2} \mathrm{PO}_{4}(1,000 \mu \mathrm{M}), \mathrm{MgSO}_{4}(500 \mu \mathrm{M})$, $\mathrm{CaCl}_{2}(1,000 \mu \mathrm{M}), \mathrm{H}_{3} \mathrm{BO}_{3}(10 \mu \mathrm{M}), \mathrm{MnSO}_{4}(0.5 \mu \mathrm{M}), \mathrm{ZnSO}_{4}$ $(0.5 \mu \mathrm{M}), \mathrm{CuSO}_{4}(0.1 \mu \mathrm{M}),\left(\mathrm{NH}_{4}\right)_{6} \mathrm{Mo}_{7} \mathrm{O}_{24}(0.1 \mu \mathrm{M})$, and Fe-EDTA $(25 \mu \mathrm{M})$. Different concentrations of $\mathrm{KNO}_{3}$ ranging from $0.2 \mathrm{mM}$ to $16 \mathrm{mM}$ were supplied to the agar medium as the nitrogen source according to the different experimental requirements, and the resulting differences in $\mathrm{K}$ concentrations were balanced by adjusting the $\mathrm{K}_{2} \mathrm{SO}_{4}$ concentration, as indicated in the figure legends. To clarify whether nitrate functions as a nutrient source to control photoperiod-modulated flowering, different concentrations of $\left(\mathrm{NH}_{4}\right)_{2} \mathrm{SO}_{4}$ including $0.2,0.5,1.0$, and $2.0 \mathrm{mM}$ were used to replace $\mathrm{KNO}_{3}$ in the agar medium, and the $\mathrm{K}$ concentrations in all $\left(\mathrm{NH}_{4}\right)_{2} \mathrm{SO}_{4}$ treatments were adjusted to $2 \mathrm{mM}$ using $\mathrm{K}_{2} \mathrm{SO}_{4}$. The $\mathrm{pH}$ was adjusted to 6.0 . The plants were transferred to a growth room where they were maintained under photoperiod cycles of $16 \mathrm{~h}$ light/ $8 \mathrm{~h}$ dark (LD) or $8 \mathrm{~h}$ light/16 $\mathrm{h}$ dark (SD). The light intensity and temperature were maintained at $100 \mu \mathrm{mol} \mathrm{m}{ }^{-2} \mathrm{~s}^{-1}$ and $22^{\circ} \mathrm{C}$, respectively. Bolting time (days after germination $[\mathrm{DAG}]$ ) and rosette leave numbers were determined when the main inflorescence had reached a height of $0.5 \mathrm{~cm}$, while flowering time (DAG) was determined when the first flower was visible.

For soil culturing, the plants were grown in square pots (6 $\mathrm{cm}$ in length and $6 \mathrm{~cm}$ in height) containing soil:perlite at a ratio of $1: 1.5$, under LD (16-h light/8-h dark) conditions. The soil and perlite were autoclaved prior to use to kill insect eggs. Seed stratification was conducted in $0.05 \%$ agar at $4^{\circ} \mathrm{C}$ for 3 days, before planting. In each pot, 4-8 wild-type seeds were sown, and a single seedling was retained at around 7 DAG. The plants were watered twice a week with approximately $20 \mathrm{~mL}$ of medium per pot, per watering event. Different concentrations of $\mathrm{KNO}_{3}$ including 1, 2, 3, 6, 10, and $20 \mathrm{mM}$ were supplied to the medium as the nitrogen source, and the resulting differences in the $\mathrm{K}$ concentrations were balanced by adjusting the $\mathrm{K}_{2} \mathrm{SO}_{4}$ concentration. The composition of other nutrients in the medium for the soil system was as follows: $\mathrm{NaH}_{2} \mathrm{PO}_{4}(1,000 \mu \mathrm{M}), \mathrm{MgSO}_{4}(500 \mu \mathrm{M}), \mathrm{CaCl}_{2}(1,000 \mu \mathrm{M})$, $\mathrm{H}_{3} \mathrm{BO}_{3}(10 \mu \mathrm{M}), \mathrm{MnSO}_{4}(0.5 \mu \mathrm{M}), \mathrm{ZnSO}_{4}(0.5 \mu \mathrm{M}), \mathrm{CuSO}_{4}$ $(0.1 \mu \mathrm{M}),\left(\mathrm{NH}_{4}\right)_{6} \mathrm{Mo}_{7} \mathrm{O}_{24}(0.1 \mu \mathrm{M})$, and Fe-EDTA $(25 \mu \mathrm{M})$.

\section{Measurement of the $\mathrm{NO}_{3}-$ Uptake}

The Arabidopsis plants were grown hydroponically for 2 weeks under either $\mathrm{LD}$ or $\mathrm{SD}$ conditions with $1 \mathrm{mM}$ nitrate as the exclusive $\mathrm{N}$ source. The nutrient solutions were renewed every second day. The daily nitrate uptake rate by the roots was then evaluated using ${ }^{15} \mathrm{NO}_{3}{ }^{-}$under the same conditions used for plant growth. Uniform plants grown on a nutrient medium containing $1 \mathrm{mM} \mathrm{KNO}_{3}$ had their medium replaced with $\mathrm{K}^{15} \mathrm{NO}_{3}$ (atom\% ${ }^{15} \mathrm{~N}, 99 \%$ ) for $24 \mathrm{~h}$, and were then harvested. The atom $\%{ }^{15} \mathrm{~N}$ was measured in an isotope ratio mass spectrometer (Isorime100; Elementar Analysensysteme, Hanau, Germany). Experiments were replicated four times for each treatment.

A non-invasive microelectrode ion flux measurement system (ipa-2; AE, United States) was used to measure the net fluxes of $\mathrm{NO}_{3}{ }^{-}$, as previously described (Hawkins et al., 2008; Zhu et al., 2019). Col-0 plants were grown in agar medium under either LD or SD conditions. Nitrate $(1 \mathrm{mM})$ was used as the exclusive $\mathrm{N}$ source and seedlings (at the stage when four leaves were visible) were used to determine the transmembrane $\mathrm{NO}_{3}{ }^{-}$fluxes of the roots in the mature zone, in the same medium used for plant growth, using a $\mathrm{NO}_{3}{ }^{-}$-selective microelectrode.

\section{Quantitative Real-Time Polymerase Chain Reaction}

The expression of $\mathrm{NO}_{3}{ }^{-}$uptake- and assimilation-related genes was determined using root samples. The expression of floweringrelated genes and stress-related marker genes was determined using the shoot samples. Approximately $100 \mathrm{mg}$ of tissue sample was ground in liquid nitrogen, and total RNA was extracted using RNAiso-Plus (TaKaRa, Otsu, Japan). The first-strand cDNA was synthesized using ReverTra Ace qPCR RT Master Mix with gDNA Remover (TOYOBO, Osaka, Japan). SYBR ${ }^{\circledR}$ Green Realtime PCR Master Mix (TOYOBO, Osaka, Japan) was then used to detect the transcript levels of the corresponding genes. The gene-specific primers used in the quantitative Real-Time PCR are listed in Supplementary Table 1. Relative transcript levels were measured, and corrected efficiency calculations were conducted, as described by Fang et al. (2016).

\section{Grafting of Arabidopsis Plants}

Grafting between the nrt1.1-1/2.1-2 mutants and Col-0 plants was performed using a previously described method (MarschMartínez et al., 2013). Briefly, plants were sown on basal media containing $1 \mathrm{mM}$ nitrate, and 5-day-old seedlings were used for grafting. After the cotyledons were removed, the hypocotyls were cut horizontally, and the scions were immediately placed into the rootstock. The successfully grafted plants were vertically placed on basal media containing $1 \mathrm{mM}$ nitrate under LD or SD conditions for flowering time measurements.

\section{Statistical Analysis}

Statistical analysis was performed using the SPSS statistical 20.0 software. One-way analysis of variance (ANOVA) with Duncan's multiple range test was used and significant differences among the treatments were indicated with $P<0.05$. Interaction differences were tested using a two-way ANOVA. The regression lines were compared using univariate analysis of variance (UNIANOVA), in which the concentrations of the nitrate were 
the covariates and the genotype (Col- 0 or $n r t 1.1-1 / 2.1-2)$ was the fixed factor, and $\triangle$ days to flowering was the dependent variable.

\section{RESULTS}

\section{Flowering Was Promoted in LD and SD Photoperiods When the Suboptimal Nitrate Supplies Were Elevated}

We first evaluated the effects of different nitrate supplies $(\sim 1 \mathrm{mM})$ on flowering in Arabidopsis thaliana ecotype Col-0 plants to clarify the effects of nitrate availability on flowering under the growth conditions used in this study. Three nitrate levels, 0.2, 1.0, and $2.0 \mathrm{mM}$, were used. As shown in Figure 1 and Supplementary Figure 1, the elevated nitrate concentrations led to earlier flowering and more rosette leaves under both LD and SD photoperiod conditions. Furthermore, the growth of the plants was improved by increasing the nitrate concentrations under both LD and SD photoperiods (Supplementary Figure 2). We then evaluated the effects of nitrate concentrations $>2 \mathrm{mM}$ on the flowering of the plants. In contrast to the above results, the days to flowering under SD conditions were delayed, but the number of rosette leaves increased with an increase in nitrate supply (Supplementary Figure 3). Under LD conditions, the flowering of the plants was also delayed when the nitrate was increased from $2 \mathrm{mM}$ to $4 \mathrm{mM}$, but further increases above $4 \mathrm{mM}$ had little effect on the delay of flowering and the number of rosette leaves. These results suggest that the flowering of Arabidopsis plants is promoted by increasing the nitrate availability, but only below an optimal level, under both LD and SD conditions. Supplementary Figure 3 clearly indicates that the optimal nitrate concentration for flowering was $\sim 2 \mathrm{mM}$ in our agar-growth system. Interestingly, a linear fit between the flowering time and nitrate concentrations of $<2 \mathrm{mM}$, generated an excellent correlation coefficient for both LD and SD conditions ( $R=0.95$ for $\mathrm{LD}$ and 0.96 for $\mathrm{SD}$ ). This illustrates the linear dependence of flowering time on nitrate availability, when its level is suboptimal for the plants, under both $\mathrm{LD}$ and SD conditions. We also measured the effects of different nitrate concentrations ( 1 to $20 \mathrm{mM}$ ) on the flowering of plants grown in a soil-based system under LD photoperiod condition. As shown in Supplementary Figure 4, the flowering time along with the elevation in nitrate supply in a soil-based system, showed a similar pattern to that in the agar-based growth system (Supplementary Figure 3). This indicated that the acceleration in flowering observed with the elevation in nitrate availability, below the optimal level, could also occur in some natural soils.

Suboptimal nitrate concentrations may cause stress to plants, and acute stresses can either delay or induce flowering (Kazan and Lyons, 2016; Takeno, 2016). To investigate this further, the expression of several stress-related marker genes, including RD29A, RD29B, KIN1, and PAP1, were determined (Supplementary Figure 5). Under both LD and SD conditions, the expression of these genes was significantly decreased when the nitrate supply was increased from $0.2 \mathrm{mM}$ to $1.0 \mathrm{mM}$, indicating that the promotion of flowering by increase of nitrate supply below $1.0 \mathrm{mM}$ may be associated with the alleviation of nitrogen-deficiency stress. Increasing the nitrate supply above $1 \mathrm{mM}$, however, had little effect on the transcript levels of these stress-related marker genes, except for KIN1 in 16-mM nitrate treatment (Supplementary Figure 5). Considering that the plants still flowered earlier in the $2-\mathrm{mM}$ nitrate treatment than in the 1-mM nitrate treatment, under both LD and SD conditions (Figure 1 and Supplementary Figures 1, 3), the floral induction
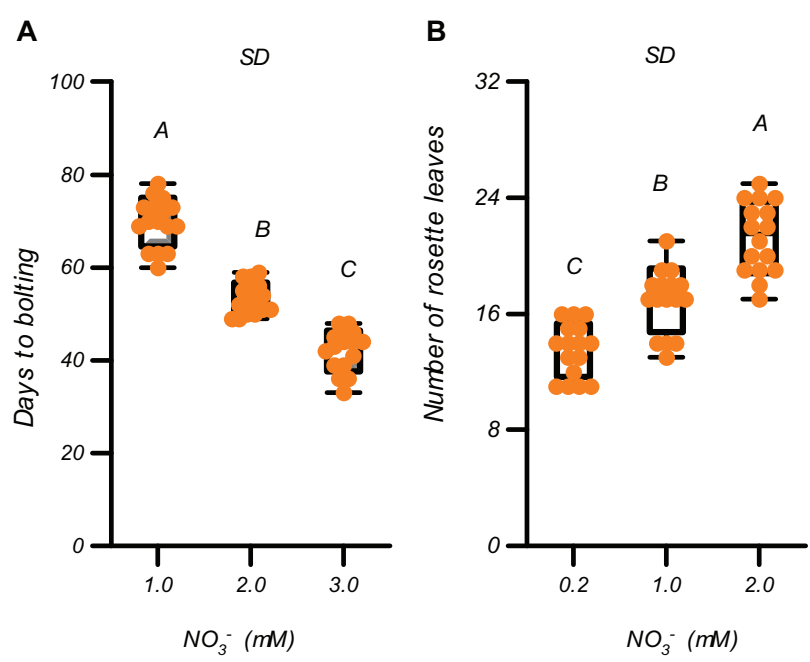

C

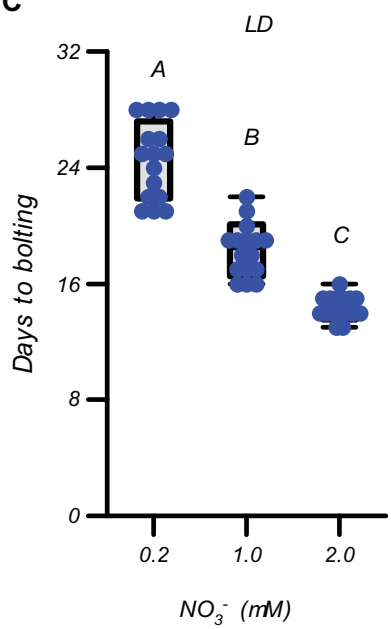

D

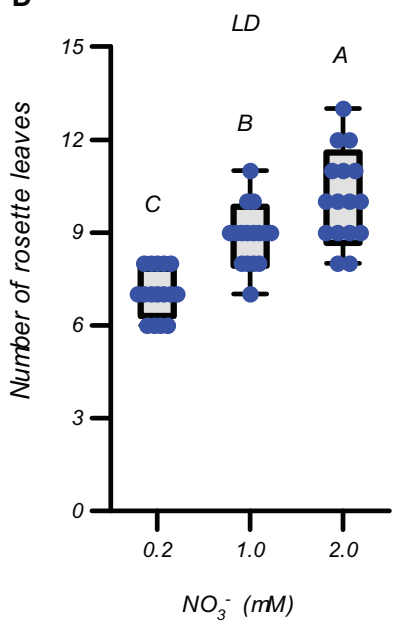

FIGURE 1 | Flowering promotion due to increases in the suboptimal nitrate supply under both LD and SD photoperiods. Days to bolting (A) and number of rosette leaves (B) of Col-0 plants grown under LD conditions (16-h light/8-h dark). Days to bolting (C) and number of rosette leaves (D) of Col-0 plants grown under SD conditions (8-h light/16-h dark). Seedlings were planted in media containing 0.2 , 1, or 2 mM nitrate. The potassium concentrations in all growth media were adjusted to $2 \mathrm{mM}$ using $\mathrm{K}_{2} \mathrm{SO}_{4}$. At least 20 plants grown in each of the LD and SD conditions were used to determine the bolting time and number of rosette leaves. Error bars represent \pm standard deviations. Different letters indicate significant differences between means, as determined using a one-way ANOVA followed by Tukey's multiple comparisons test $(P<0.05)$. 
from increasing the suboptimal nitrate supply, may not be solely associated with the alleviation of nitrogen-deficiency stress.

\section{LD Induction of Flowering Is Associated With Nitrate Availability}

As flowering is promoted by the elevation in nitrate availability below an optimal level under both LD and SD conditions, we investigated whether the nitrate availability in the growth medium affected the photoperiodic flowering response. Two-way ANOVAs are reported as an effective way to test the interactions between these two treatments (Brady et al., 2015) and were consequently used for data analysis as shown in Figures 1A,C. In addition to the unilateral impact of the photoperiod or nitrate availability, a significant interaction between these two environmental factors was also observed in the control of the flowering time of the plants, that is, the $P$-value of the model term "photoperiod $\times$ nitrate" was $1.36 \mathrm{E}^{-22}$ (Figure 2A). Therefore, we investigated how nitrate availability affects the photoperiodic flowering responses in plants. By subtracting the bolting time of the plants grown under LD conditions from those grown under SD conditions, within the same nitrogen treatment, a time interval for bolting ( $\triangle$ days to bolting), defined as the time of LDinduced flowering (TLIF), was generated. The results showed that the increases in the nitrate supply clearly minimized the TLIF (e.g., TLIF $=50.2 \mathrm{~d}$ in $0.2-\mathrm{mM}$ nitrate treatment and $29.1 \mathrm{~d}$ in 2-mM nitrate treatment). These results indicated that flowering under SD conditions could partially mimic the flowering under
LD conditions if the nitrate availability was improved. We also fitted the TLIF and the level of nitrate supply data with a linear model and found that the TLIF was negatively correlated with the level of nitrate supply $(R=0.9828$, Figure $2 B$ ), thus providing further support for the above conclusions.

In addition to being an essential nutrient (i.e., nitrogen source) for plant growth and development, nitrate also serves as a signaling molecule that regulates several physiological processes (Alboresi et al., 2005; Konishi and Yanagisawa, 2013; Krapp et al., 2014; Bouguyon et al., 2015; Maeda et al., 2018; Olas et al., 2019). To clarify how nitrate functions as a nitrogen source to control photoperiod-modulated flowering, we tested the TLIF of Col-0 plants grown with ammonium as an alternative $\mathrm{N}$ source (the individual LD and SD data sets are shown in Supplementary Figure 6). The results showed that the TLIF was linearly correlated when the level of ammonium supplied was ranged from 0.2 to $2 \mathrm{mM}$ ( $R=0.9906$ Figure $2 \mathrm{~B}$ ), indicating that the nitrogen-source action of the nitrate may be a part of the nitrate mechanism for controlling the TLIF. However, when comparing this TLIF-ammonium regression line with the above TLIF-nitrate regression line, using a UNIANOVA, we found that the decrease in the TLIF in response to the elevated nitrate supply was significantly steeper than in response to the elevated ammonium supply. This difference could also be seen by comparing the slopes of the above two regression lines $(-11.890$ and -5.67 for TLIF-nitrate and TLIF-ammonium regression lines, respectively) (Figure $2 \mathbf{B}$ ). We then analysis the flowering of nia2-5 mutant, which has less than $10 \%$ of

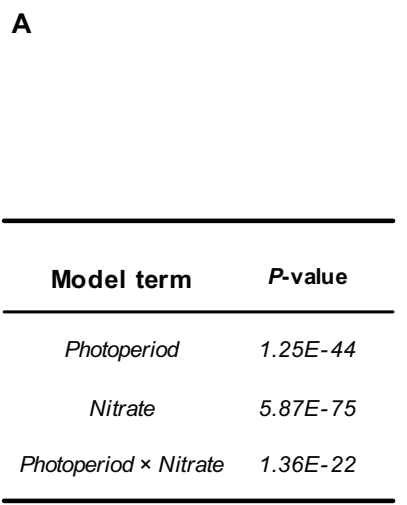

\section{B}

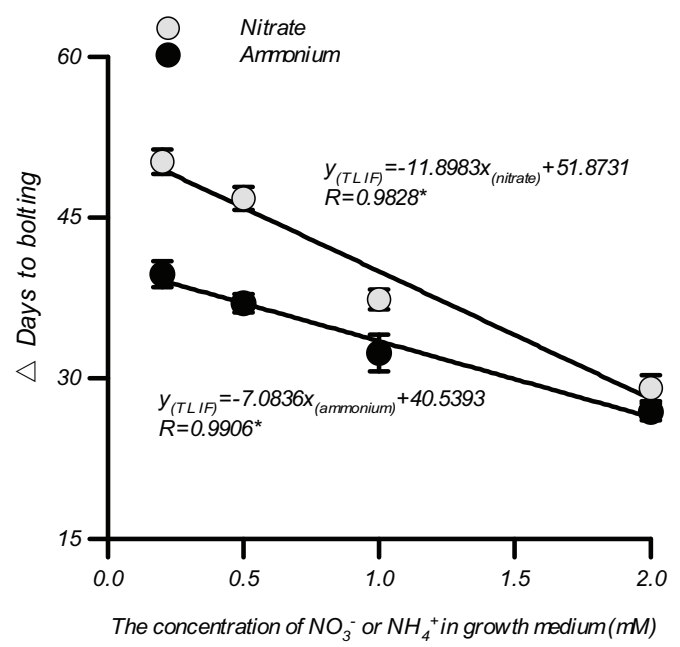

C

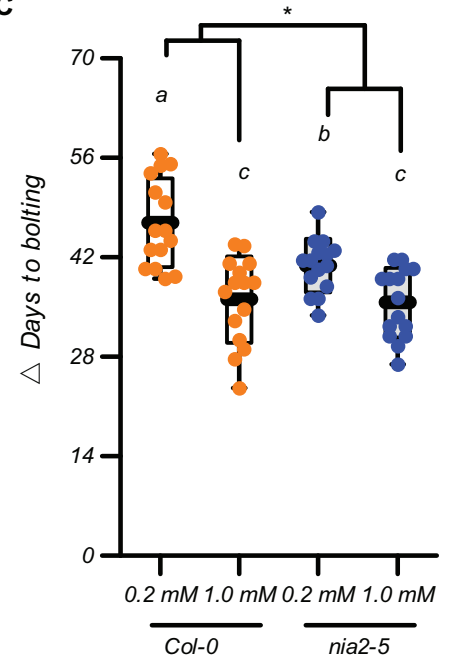

FIGURE 2 | Association of flowering under LD conditions with nitrate availability. (A) Statistical analysis of the data from Figures 1A,C. Data were analyzed using a two-way ANOVA followed by Duncan's multiple range test. (B) The time of LD-induced flowering ( $\triangle$ days to bolting) in the Col-0 plants grown with different nitrogen sources. Different concentrations of $\left(\mathrm{NH}_{4}\right)_{2} \mathrm{SO}_{4}$ including $0.2,0.5,1.0$, and $2.0 \mathrm{mM}$ were used to replace the $\mathrm{KNO}_{3}$ in the agar medium. (C) The time of $\mathrm{LD}$-induced flowering ( $\triangle$ days to flowering) in the Col-0 plants and nia2-5 mutants, grown with $0.2 \mathrm{mM}$ or $1 \mathrm{mM}$ nitrate. Seedlings were grown on agar media containing nitrate or ammonium as indicated. The potassium concentrations in all growth media were adjusted to $1 \mathrm{mM}$ using $\mathrm{K}_{2} \mathrm{SO}_{4}$. LD: 16-h light/8-h dark; SD: 8-h light/16-h dark. $\triangle$ Days to bolting was calculated by subtracting the bolting time of plants grown under LD conditions from that of plants grown under SD conditions, with the same nitrogen treatment. Raw data of panels $\mathbf{B}, \mathbf{C})$ from individual photoperiods are shown in Supplementary Figures 6, 7. At least 20 plants grown in each of the LD and SD conditions were used for the quantification of flowering. Error bars represent \pm standard deviations. Different letters indicate significant differences between means, as determined using a two-way ANOVA followed by Tukey's multiple comparisons test $(P<0.05)$. Asterisk indicates significant interaction of photoperiod $\times$ nitrogen source $\mathbf{( B )}$ or photoperiod $\times$ genotype $\mathbf{( C )}$ at $P<0.05$ (two-way ANOVA). 
the wild-type nitrate reductase activity (Desikan et al., 2002; Wang et al., 2004; Seligman et al., 2008), was used for further investigations. As shown in Figure 2C, in comparison with that in the Col-0 plants, the difference in TLIF between the $0.2 \mathrm{mM}$ and $1 \mathrm{mM}$ nitrate treatments was clearly diminished in the nia2-5 mutants, suggesting that the sensitivity of the photoperiodic flowering responses to elevated levels of nitrate were also reduced by the dysfunction of the nitrate assimilation pathway. Moreover, the increase in nitrate supply still led to a statistically significant decrease in TLIF in the nia2-5 mutants (Figure 2C and Supplementary Figure 7). Collectively, these results indicated that in addition to the nitrate's nutritional function, it may also function as a signaling-molecule to induce flowering during extended photoperiods.

\section{LD Photoperiods Upregulate Nitrate Uptake by NRT1.1/NRT2.1}

We investigated how the photoperiod affects root nitrate uptake in plants. The daily nitrate uptake rates by the roots of the Col-0 plants were compared between the LD and SD treatments. The plants were hydroponically pre-cultured with an intermediate concentration of nitrate $(1 \mathrm{mM})$, under either $\mathrm{LD}$ or $\mathrm{SD}$ conditions. The daily nitrate uptake rates by the roots were then evaluated using ${ }^{15} \mathrm{NO}_{3}{ }^{-}$under the same conditions used for the plant growth. The results showed that they increased by about 1.6-fold with the LD treatment when compared to the SD treatment (Figure $\mathbf{3 A}$ ). The non-invasive micro-test technique, which uses computer-controlled stepper motors to oscillate an ion-selective microelectrode near the surfaces of the tissues, could provide a real-time measurement of the ion flux based on Fick's first law of diffusion (Shabala et al., 2016). Therefore, this technique was used to continuously monitor the transmembrane $\mathrm{NO}_{3}{ }^{-}$fluxes in the roots over a 24-h period. The maturation zone in roots has a greater net nitrate influx than the other root zones and this root zone makes up most of the root surface area (Zhu et al., 2019). Therefore, the transmembrane $\mathrm{NO}_{3}{ }^{-}$fluxes of the roots in the maturation zones were monitored in this study, to investigate the effects of photoperiod on root nitrate uptake. Although the net nitrate influx in the roots was observed under dark conditions, it was extremely low with both LD and SD treatments (Figures 3B,C). In accordance with earlier studies (Delhon et al., 1995; Peuke and Jeschke, 1998), the net nitrate influx of the roots was greatly increased under the light conditions, when compared with the dark conditions (Figures 3B,C). Interestingly, the LD treatments resulted in a higher net nitrate influx than the SD treatments, despite the plants being illuminated during the first $8 \mathrm{~h}$ per day under both photoperiod conditions (Figure 3B). In addition, the cumulant calculation showed that the net nitrate influx increased by $\sim 35 \%$ with the LD treatment, when compared with the SD treatment (Figure 3C). These results suggest that both a longer illumination time and a higher nitrate uptake rate during the illumination period contributed to the improved nitrate uptake under LD conditions.

Currently, six nitrate transporters (NRTs) have been characterized to be involved in root nitrate uptake in Arabidopsis plants. Among these, NRT1.1 and NRT2.1 play the most pivotal roles in root nitrate uptake in most growth environments ( $\mathrm{Li}$ et al., 2006), whereas NRT1.2, NRT2.2, NRT2.4, and NRT2.5 only contribute to minor levels of root nitrate uptake (Wang et al., 2012; Lezhneva et al., 2014). The expression of NRT1.1 and NRT2.1 in the roots was analyzed to understand how these two NRTs act to control root nitrate uptake under different photoperiod conditions. As shown in Figures 4A,B, these two genes were markedly upregulated during the day-time in both

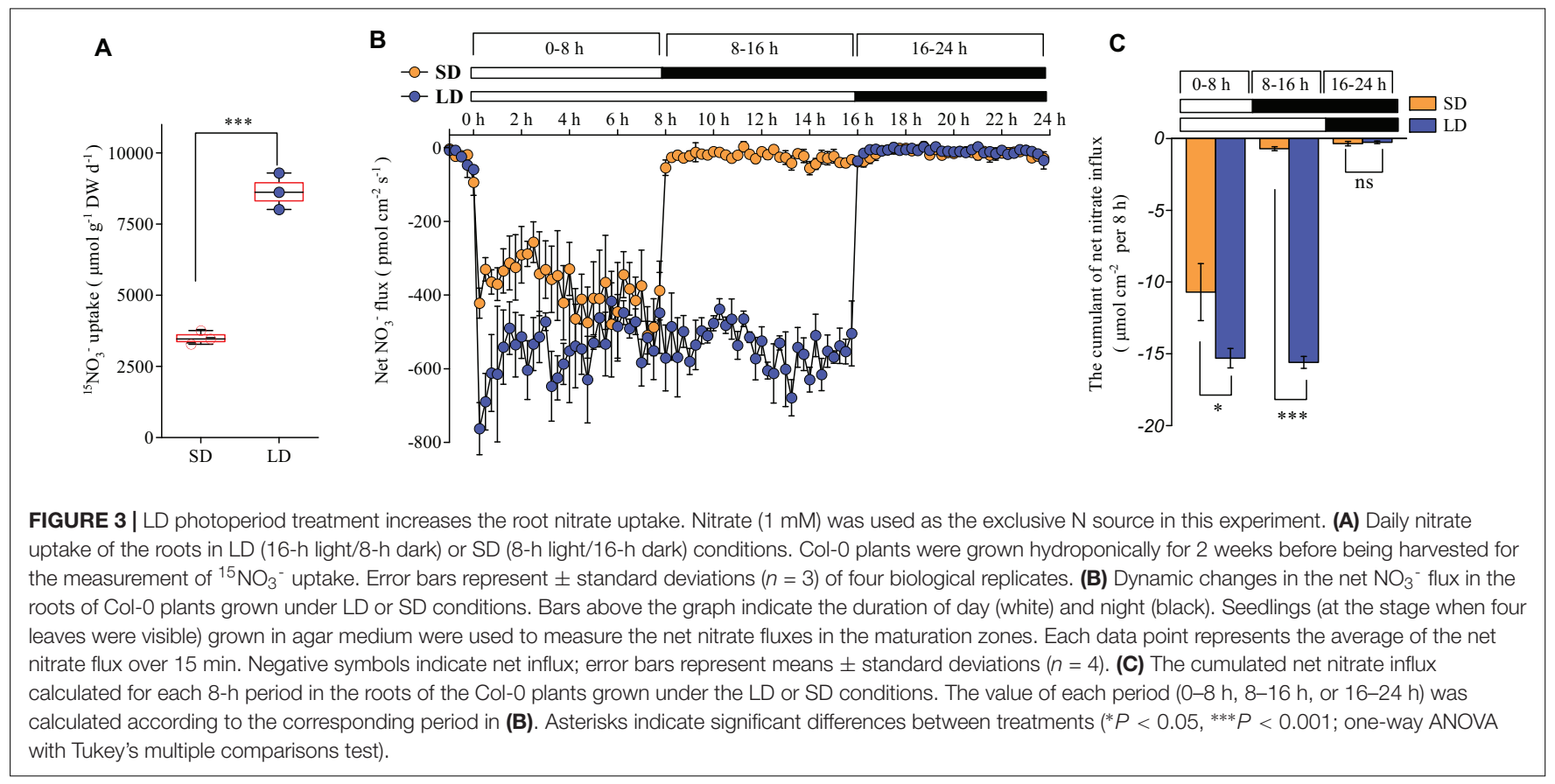


LD- and SD-grown plants, but the up-regulation of both genes was much higher at the peak time point in the LD-grown plants (ZT $16 \mathrm{~h}$ for NRT1.1 and ZT $12 \mathrm{~h}$ for NRT2.1) than in the SD-grown plants (ZT $8 \mathrm{~h}$ for NRT1.1 and ZT $4 \mathrm{~h}$ for NRT2.1). As shown in Supplementary Figure 8, NRT2.2, NRT2.4, and NRT2.5 were differentially regulated by the photoperiod, and their expression was much lower than that of the NRT1.1 and NRT2.1 (Figure 4). In addition, the expression of NRT1.2 was barely affected by the photoperiod. These results, together with the fact that NRT1.1 and NRT2.1 are crucial for root nitrate uptake under most conditions (Li et al., 2006; Wang et al., 2012; Lezhneva et al., 2014), indicated that higher expression levels of NRT1.1 and NRT2.1 in the roots of the LD-grown plants may be the most important factor leading to the higher root nitrate uptake when compared with the SD-grown plants. The nrt1.11/nrt2.1-2 double mutant, which possessed T-DNA insertions in the exon and intron of the NRT1.1 and NRT2.1 genes, respectively, were then used to clarify the above speculations. Although the T-DNA insertion in the intron of the NRT2.1 gene also leads to the deletion of its adjacent gene NRT2.2 (Li et al., 2006; Ye et al., 2019), the effect of this deletion on the root nitrate uptake could be limited in our present growth conditions for the nrt1.1-1/nrt2.1-2 double mutant, because the contribution of NRT2.2 to the root nitrate uptake was very small (Li et al., 2006). In this study, we found that the loss of action of NRT1.1/NRT2.1 in the $n r t 1.1-1 / n r t 2.1-2$ mutants substantially inhibited the daily nitrate uptake rate (Figures 4C,D). Furthermore, the two-way ANOVA analysis revealed that the LD increased the daily nitrate uptake rate in the $n r t 1.1-1 / n r t 2.1-2$ mutants, which was much lower than that in the Col-0 plants. Collectively, these results suggested that the LD-improved nitrate uptake by roots may be associated with the up-regulation of NRT1.1 and NRT2.1.

\section{LD-Induced Flowering Is Associated With the Action of NRT1.1/NRT2.1}

Given that the LD conditions improved the root nitrate uptake by affecting the NRT1.1 and NRT2.1, we aimed to clarify our previous speculation that photoperiodic flowering responses may be associated with alternative nitrate uptake. We first compared the bolting time of the nrt1.1-1 mutants, the nrt2.1-2 mutants, and the nrt1.1-1/2.1-2 mutants in a soil system. As shown in Supplementary Figure 9, both $n r t 1.1-1$ and the $n r t 2.1-2$ bolted later than Col-0, but earlier than the nrt1.1-1/2.1-2 mutants. Furthermore, the nrt1.1-1/2.1-2 mutants had a higher TLIF than that of $n r t 1.1-1$ and $n r t 2.1-2$. This result indicated that NRT1.1 and NRT2.1 were additively involved in LD-induced flowering. Therefore, the flowering responses of the Col- 0 plants and the nrt1.1-1/nrt2.1-2 mutants were further compared using different photoperiod treatments in the agar medium, with $1 \mathrm{mM}$ nitrate. Figure 5A shows that the main inflorescence of the Col-0 plants reached a height of approximately $2 \mathrm{~cm}$ on the 22nd and 54th day of growth under the LD and SD treatments, respectively, but nrt1.1-1/nrt2.1-2 mutants had not yet bolted in both treatments. We then analyzed the TLIF of the Col-0 plants and nrt1.1-1/2.1-2 mutants in the media with different nitrate supplies (the individual LD and SD data sets are shown in Supplementary Figure 10). Although the TLIF of the $n r t 1.1-1 / n r t 2.1-2$ mutants was also linearly and negatively correlated with nitrate concentrations below $2 \mathrm{mM}$ $(R=0.9947$, Figure 5B), the slope of the regression line was significantly smaller than that of the Col-0 plants (UNIANOVA, $P$-value $<0.0001)$, which suggested that the loss of action of the NRT1.1/NRT2.1 in the $n r t 1.1-1 / 2.1-2$ mutants reduced the sensitivity of the photoperiodic flowering responses to the elevated levels of nitrate. Moreover, the TLIF in the nrt1.11/2.1-2 mutants, which mimicked the TLIF of the Col-0 plants
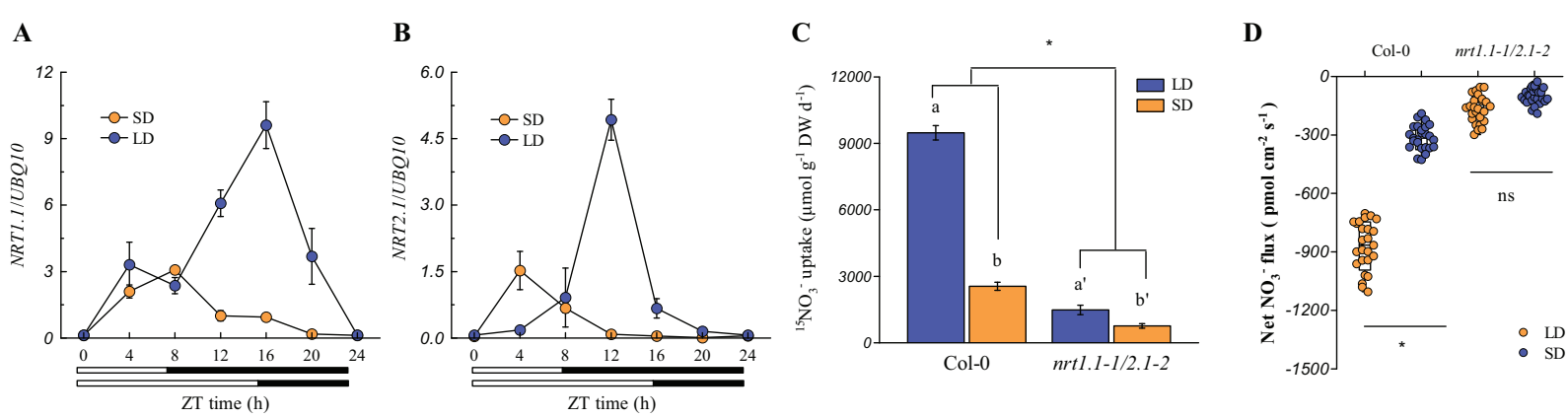

FIGURE 4 | LD photoperiods upregulate NRT1.1/NRT2.1-mediated nitrate uptake in the roots. The expression pattern of NRT1.1 (A) and NRT2.1 (B) in the roots of Col-0 plants grown under LD (16-h light/8-h dark) and SD (8-h light/16-h dark) photoperiods. Seedlings (at the stage when four leaves were visible) grown in agar medium with $1 \mathrm{mM} \mathrm{KNO}_{3}$ were harvested. Bars below the graph indicate the duration of day (white) and night (black). Expression levels are normalized to the expression level of UBIQUITIN 10. (C) Nitrate uptake rate in the roots of the Col-0 plants and nrt1.1-1/2.1-2 mutants under both LD and SD conditions. The plants were grown hydroponically for 2 weeks with $3 \mathrm{mM} \mathrm{KNO}_{3}$ to ensure that the nrt1.1-1/2.1-2 plants would not show severe signs of $\mathrm{N}$ limitations. The plants were then grown in $1 \mathrm{mM}$ nitrate for another week, after which the daily nitrate uptake rate by the roots was evaluated using $1 \mathrm{mM}^{15} \mathrm{NO}_{3}{ }^{-}$. (D) Supplementary Figure $14 \mathrm{Net}$ $\mathrm{NO}_{3}{ }^{-}$flux in the roots of Col-0 plants and nrt1.1-1/2.1-2 mutants grown in agar systems under SD and LD conditions. Nitrate (1 mM) was used as the exclusive $\mathrm{N}$-source in this section. Seedlings (at the stage when four leaves were visible) grown in agar medium were used to measure the net nitrate fluxes in the maturation zones at ZT 1-2 h. Data represent the means \pm standard deviations $(n=4)$. Different letters indicate significant differences between the means as determined by using a two-way ANOVA followed by Tukey's multiple comparisons test $(P<0.05)$. Significant interactions between the photoperiod and genotype are indicated by an asterisk ( ${ }^{*} P<0.05$; ns, non-significant). 
A

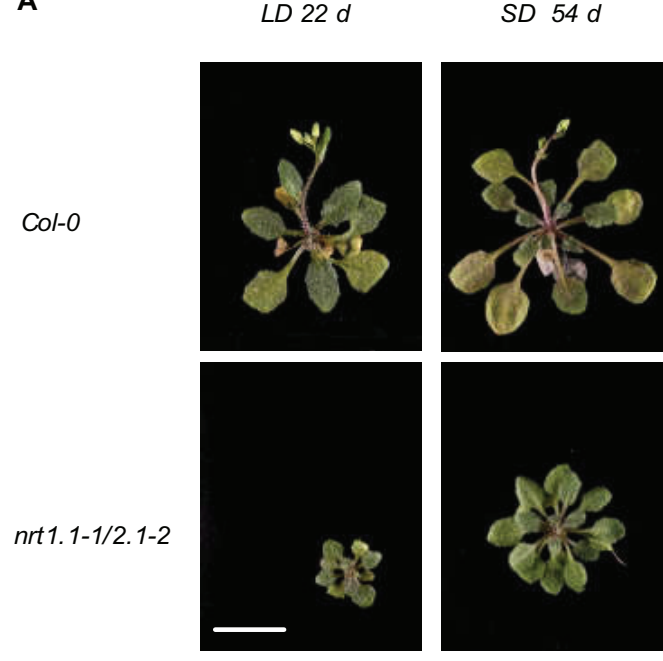

B

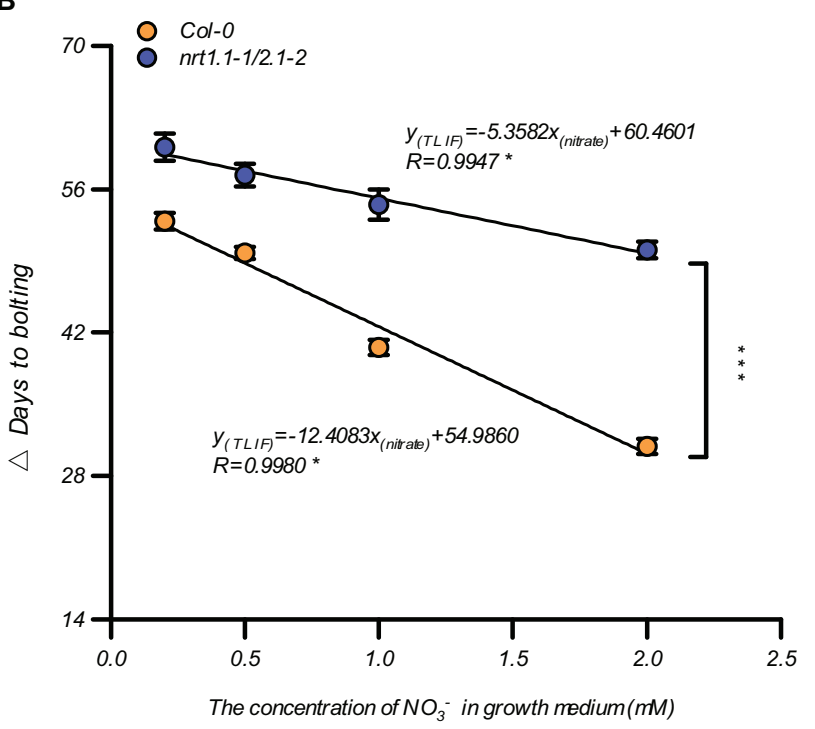

FIGURE 5 | NRT1.1- and NRT2.1-mediated nitrate uptake confers LD-induced flowering. (A) Phenotypic photographs of Col-0 and nrt1.1-1/2.1-2 plants treated with $1.0 \mathrm{mM}$ nitrate. The plants were grown in an agar system containing nitrate as the sole $\mathrm{N}$ source, under SD and LD conditions. Scale bar = $1 \mathrm{~cm}$. (B) The time of the LD-induced flowering ( $\Delta$ days to bolting) in the Col-0 plants and nrt1.1-1/2.1-2 mutants. Seedlings were grown on agar medium containing a series of $\mathrm{KNO}_{3}$

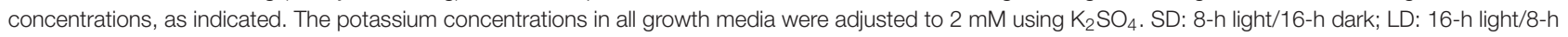
dark. $\Delta$ Days to bolting was calculated by subtracting the bolting time of the plants grown under LD conditions from that of the plants grown under SD conditions, with the same nitrogen treatment. Raw data from individual photoperiods are shown in Supplementary Figure 10. At least 20 plants grown in each of the LD and SD conditions were used for the quantification of flowering. Error bars represent \pm standard deviations $(n=12)$. The regression lines were compared using a UNIANOVA. Significant differences between two regression lines were indicated by an asterisk $\left.{ }^{\star \star \star} P<0.0001\right)$.

in the lower nitrate treatments, was significantly increased, compared with that of the wild-type plants with the same nitrate concentrations. Collectively, these results indicate that the actions of the NRT1.1/NRT2.1 were involved in the LDinduced flowering.

Given that NRT1.1 and NRT2.1 are also expressed in the shoots (Guo et al., 2001; Okamoto et al., 2003), the role of NRT1.1/NRT2.1 is clearly not limited to nitrate uptake in the roots. We aimed to determine whether NRT1.1/NRT2.1 only confers LD-induced flowering by affecting nitrate uptake in the roots or by also affecting it in their shoot-part functions. Therefore, we reciprocally grafted $n r t 1.1-1 / 2.1-2$ shoot scions onto wild-type rootstocks ( $n r t 1.1 / 2.1 / \mathrm{Col}-0)$ and wild-type shoot scions onto $n r t 1.1-1 / 2.1-2$ rootstocks (Col-0/nrt1.1/2.1), and determined the flowering time of the grafted combinations. Plants were also homo-grafted as controls (Col-0 scion/Col-0 stock and nrt1.1-1/2.1-2 scion/nrt1.1-1/2.1-2 stock). As shown in Figure 6, the TLIF of the $n r t 1.1-1 / 2.1-2$ rootstocks grafted with the Col-0 shoots (Col-0/nrt1.1/2.1) was significantly higher than that of the self-grafted wild-type plants (the individual LD and SD data sets are shown in Supplementary Figure 11). Given the above finding that the LD conditions upregulated the nitrate uptake of the NRT1.1/NRT2.1, these results suggested that root nitrate uptake was controlled by NRT1.1/NRT2.1 and should be involved in LD-induced flowering. This assumption was further supported by the observation that TLIF was significantly decreased in the shoots of nrt1.1-1/2.1-2 grafted with the Col0 rootstocks.
Interestingly, we also found that the TLIF of the $n r t 1.1 / 2.1 /$ Col-0 grafts was significantly increased compared with that of the Col-0/Col-0 grafts. These results suggest that the shoot-part function of the NRT1.1/NRT2.1 was also involved in the LD-induced flowering. It is noteworthy that the TLIF of the $n r t 1.1 / 2.1 / \mathrm{Col}-0$ grafts was even greater than that of the Col-0/nrt1.1/2.1 grafts, indicating that the shoot-part function of the NRT1.1/NRT2.1 may contribute more to the LD-induced flowering than the root-part functions.

\section{Improved Nitrate Status in the LD-Induced Flowering Was Associated With the Up-Regulation of Flowering-Related Genes}

In most cases, flowering induced by the LD photoperiod was under the control of the canonical genetic GI-CO-FT pathway (Fornara et al., 2010; Tiwari et al., 2010; Andrés and Coupland, 2012; Yuan et al., 2016; Luo et al., 2018; Bao et al., 2019). Therefore, we first investigated whether the nitrate availability at different concentrations affected the expression of several key genes involved in this pathway, including GI, FKF1, COP1, $C O$, and FT. The plants at the four-leaf visible stage were used to investigate gene expression. As shown in Supplementary Figure 12, only the expression of $C O$ and $F T$ were upregulated dramatically in the Col-0 plants with $1 \mathrm{mM}$ nitrate when compared to the $0.2-\mathrm{mM}$ nitrate treatments under LD conditions; in contrast, the loss of function of NRT1.1/NRT2.1 in the 


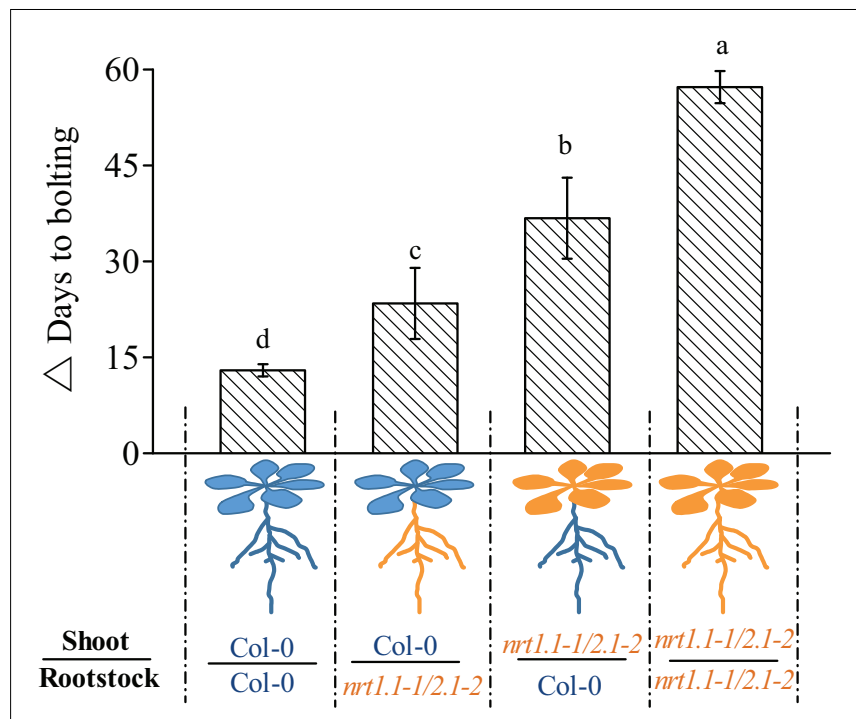

FIGURE 6 | Time of LD-induced flowering in grafted plants under $1 \mathrm{mM}$ nitrate conditions. $\triangle$ Days to bolting was determined by subtracting the bolting time of the plants grown under LD conditions from that of the plants grown under SD conditions. Raw data from the individual photoperiods are shown in Supplementary Figure 11. Treatments are similar to those presented in Figure 3. Bars represent means \pm standard deviations $(n=6)$. Different letters above the bars indicate significant differences between the means, as determined using a one-way ANOVA followed by Tukey's multiple comparisons test $(P<0.05)$.

nrt1.1-1/2.1-2 mutants strongly inhibited this increase. We then investigated the temporal expression $(5,7,9$, and 11 days after germination [DAG]) of FT and $C O$ in the shoots of the seedlings treated with the different nitrate concentrations under LD conditions (Figure 7). The results showed that at 5 DAG, the expression of both genes was not significantly different from the different nitrate supplies. However, the elevation in nitrate supply increased the expression of the CO and FT from 7 and 9 DAG, respectively. Similar to the results observed above, the differences in the expression of these two genes was clearly reduced by the loss of function of NRT1.1/NRT2.1, indicating that the induction of CO and FT with the LD photoperiod was associated with an improved nitrate status in plants. Subsequently, we compared the flowering responses between the Col-0 plants and the co and $f t$ mutants under LD conditions. As shown in Figure 8 and Supplementary Table 2, the elevation in the nitrate levels had a smaller effect on the promotion of flowering in both $\mathrm{co}-1$ and $f t-10$ mutants, when compared with the Col-0 plants. Taken together, the contributions of the improved nitrate status to the LD photoperiod-induced flowering is partially associated with the upregulation of $C O$ and FT expression.

In addition to the CO-FT pathway, Yuan et al. (2016) indicated that the blue-light receptor cryptochrome 1 (CRY1) also played a critical role in the $\mathrm{N}$-regulated flowering of Arabidopsis. Considering that the elevation in nitrate supply were found to increase CRY1 expression (Supplementary Figure 12G), the contribution of the improved nitrate status to the up-regulation of CRY1 may also play a role in LD-induced flowering. Therefore, we also determined the effects of nitrate availability below the optimal level for the flowering of CRY1 loss-of-function mutants under both SD and LD conditions (Supplementary Table 3). The results showed that increasing the nitrate concentration had no significant effect on the promotion of flowering in the cry 1 mutants, under either LD or SD conditions, supporting the above assumption. Interestingly, the elevation in nitrate concentrations also increased the number of rosette leaves in the cry 1 mutants, but this increase was less than that observed in the Col-0 plants (Supplementary Table 3). Therefore, the process by which nitrate affects plant vegetative growth might be partially associated with CRY1.

\section{DISCUSSION}

To ensure reproductive success and seed production, the flowering of plants is controlled by sophisticated regulatory networks that monitor changes in the environment, especially changes in the photoperiod (Andrés and Coupland, 2012; Yuan et al., 2016; Luo et al., 2018; Bao et al., 2019, 2020). In this study, we have reported that nitrate play a role in LD-induced flowering. We found that when the nitrate availability in the growth system was below the optimal level for the flowering of plants, the LD conditions improved the nitrate status by upregulating NRT1.1/NRT2.1 expression, which subsequently upregulates the expression of light-responsive regulators FT and $\mathrm{CO}$ in plants, thereby modulating the timing of floral induction.

As demonstrated previously, and in this study, the elevation in nitrate availability in the growth system below an optimal level resulted in a clear acceleration of the flowering process (Lin and Tsay, 2017; Figure 1 and Supplementary Figure 1), which decreased the difference in flowering time between the LD and SD conditions (Figure 2 and Supplementary Figure 3). This suggested that the photoperiodic flowering response is associated with the nitrate status in Arabidopsis plants, and that flowering under LD conditions could be partially mimicked under SD conditions if nitrate concentrations were elevated. As an essential nutrient for plants, nitrate can greatly affect plant growth and development, such as seed germination, shoot branching, lateral root formation, and flowering (Alboresi et al., 2005; Castro Marín et al., 2011; Bouguyon et al., 2015; Zeng et al., 2017). Previous studies have indicated that the number of rosette leaves at flowering is related to the days to flowering under most conditions when there is a sufficient supply of nitrate (Koornneef et al., 1991; Bagnall, 1993; Pouteau et al., 2004; Pouteau and Albertini, 2009; Lin and Tsay, 2017; Gras et al., 2018). Therefore, the number of rosette leaves at flowering is often used as an indicator of floral transition under most conditions when there is a sufficient supply of nitrate. However, in an agar-based growth system with suboptimal nitrate concentrations, we found that the flowering time was negatively correlated with the number of rosette leaves and biomass production under both LD and SD conditions (Figure 1 and Supplementary Figures 1, 10). Of note, in a soil-based growth system, Lin and Tsay (2017) also found that plants grown in $1.0 \mathrm{mM}$ nitrate had shorter flowering times and more rosette leaves than plants grown in $0.4 \mathrm{mM}$ 
A

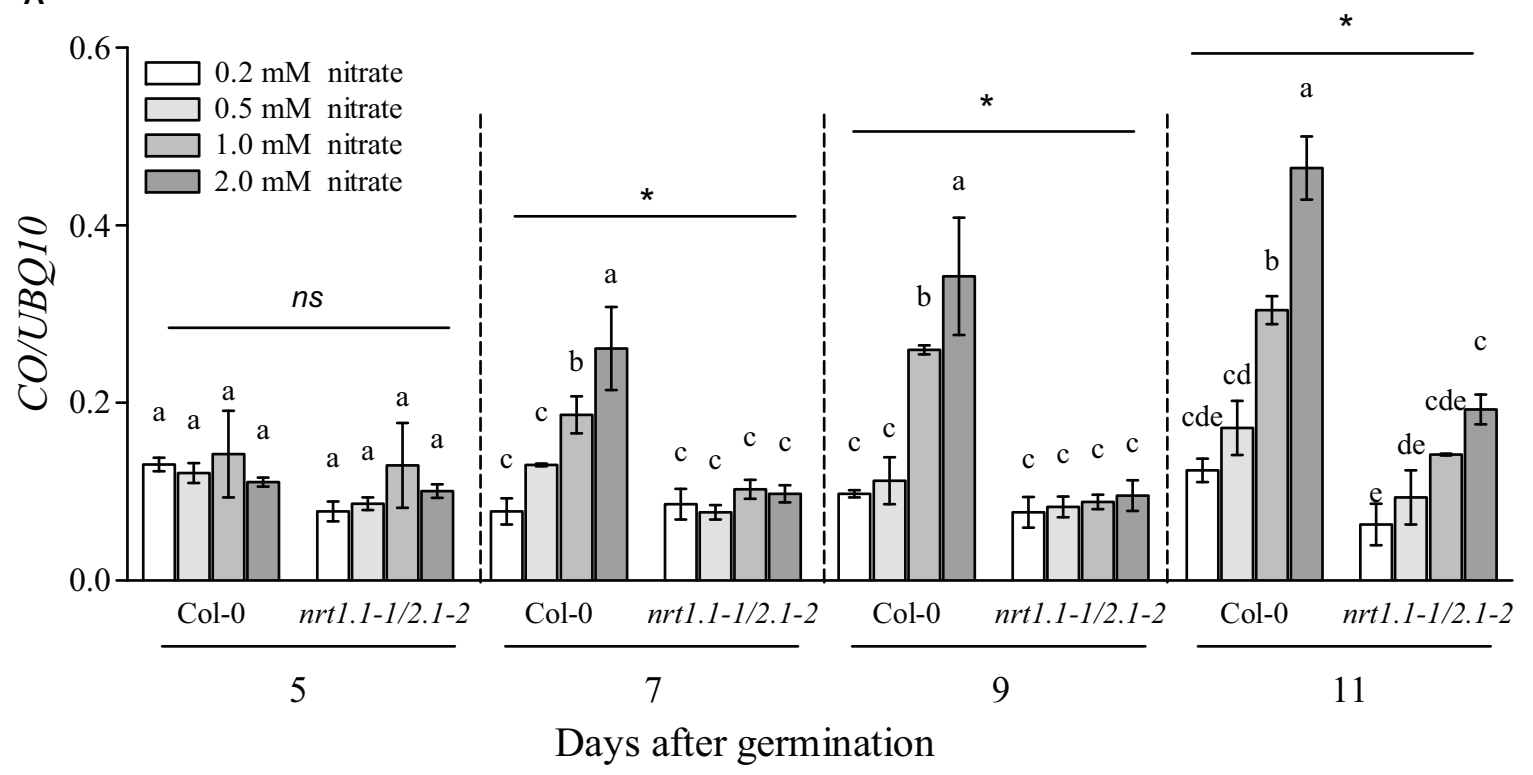

B

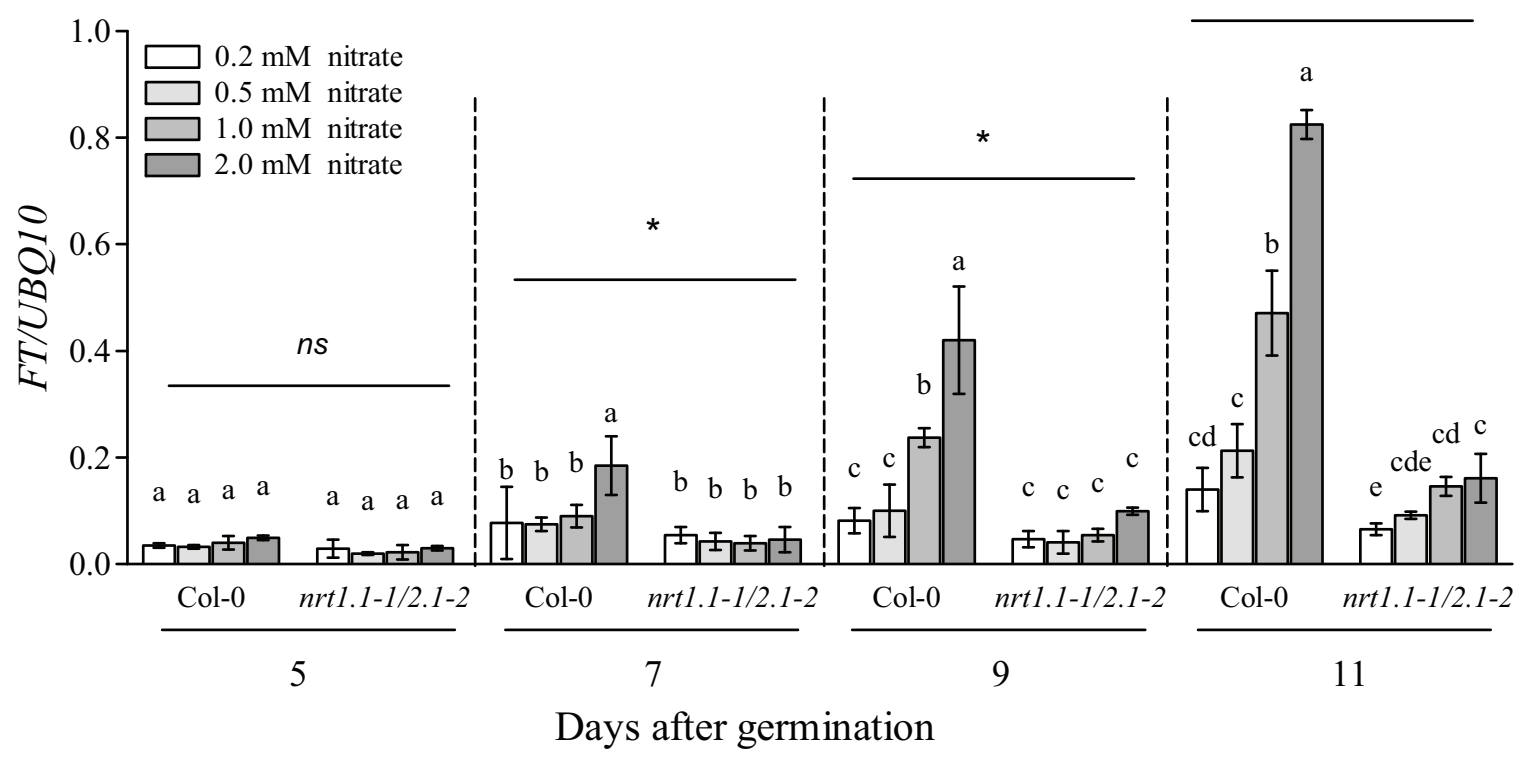

FIGURE 7 | Loss of function of NRT1.1 and NRT2.1 suppressed the induction of $F T$ and CO by increasing nitrate supplies under LD conditions. Temporal expression of $C O(\mathbf{A})$ and $F T(\mathbf{B})$ in the shoots of Col-0 and nrt1.1-1/2.1-2 plants grown in 0.2, 0.5, 1.0, and 2.0 mM nitrate treatments, under LD conditions. Plants were harvested at zeitgeber time (ZT) $16 \mathrm{~h}$ for RNA extraction, after the indicated number of germination days. Potassium concentrations in all growth media were adjusted to $2 \mathrm{mM}$ using $\mathrm{K}_{2} \mathrm{SO}_{4}$. Expression levels are normalized to the expression level of UBIQUITIN 10. Bars represent means \pm standard deviations ( $n=4$ ). Different letters indicate significant differences between the means, as determined using a two-way ANOVA followed by Tukey's multiple comparisons test $(P<0.05)$. Significant interactions between photoperiod and genotype are indicated by an asterisk ( ${ }^{\star} P<0.05$; ns, non-significant).

nitrate. We assumed that the floral transitions below the optimal nitrate concentrations may not merely be a consequence of the cessation of leaf production, and that other factors affecting the quantitative responses might also be involved. The shortage of nitrogen for leaf production under low nitrate conditions may explain the aforementioned results. However, more studies are required to draw clear conclusions. We also found that the acceleration in flowering observed with the elevation in nitrate availability, below the optimal level, could also occur in some natural soils (Supplementary Figure 4). However, the optimal nitrate level for promoting flowering in the soil system is higher than that in the agar system (Supplementary Figures 3, 4). Therefore, although the results observed in the agar system could help predict results in the soil system, caution should be exercised when directly applying these results in natural systems. Notably, the promotion of flowering and the increase in the 
A

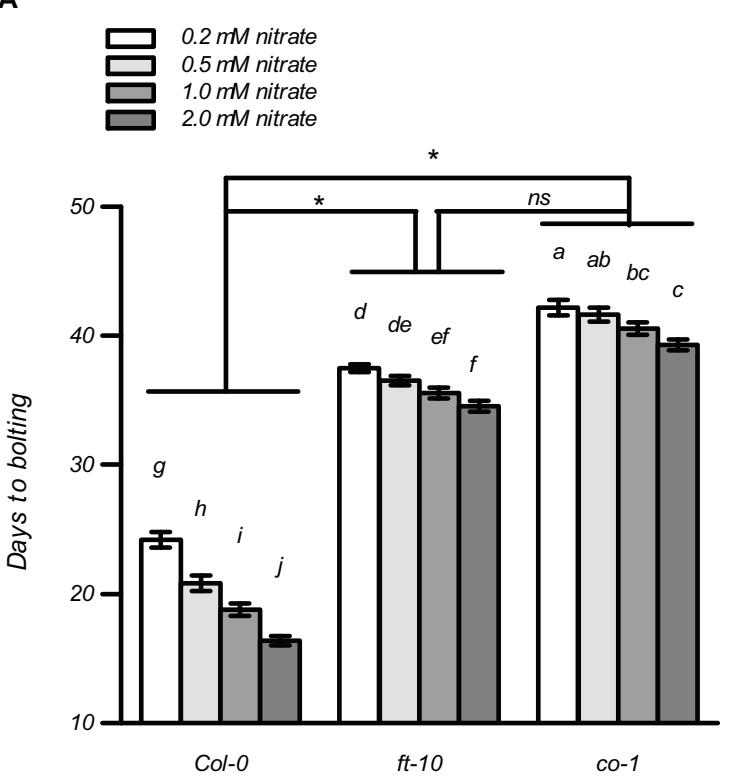

B
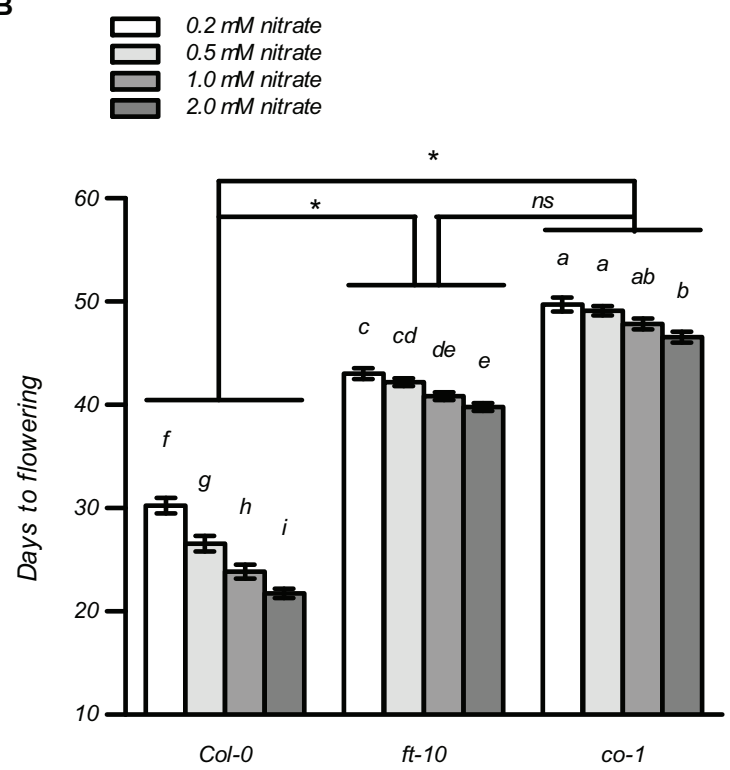

FIGURE 8 | Effects of nitrate availability on flowering in $\mathrm{ft}-10$ and co-1 mutants under LD conditions. Days to bolting (A) and days to flowering (B) of the plants grown with different nitrate concentrations, were determined in an agar system under LD conditions (16 h light/8 h dark). Bars represent means \pm standard deviations $(n=20)$. Different letters indicate significant differences between the means, as determined using a two-way ANOVA followed by Tukey's multiple comparisons test $(P<0.05)$. Significant interactions between the photoperiod and genotype are indicated by an asterisk $\left({ }^{*} P<0.05\right.$; ns, non-significant).

number of rosette leaves resulting from the elevation in nitrate concentrations under SD conditions were more significant than those under LD conditions (Supplementary Figure 3). A similar result was also observed in the investigation by Castro Marín et al. (2011). This is probably because growth is much slower and the vegetative period is extended under SD conditions that the effect of nitrate availability on plant vegetative growth becomes more prominent. Furthermore, although nitrate at high concentrations $(>4 \mathrm{mM}$ ) did not alter flowering time, nitrate below the optimal level did promote plant growth and the flowering of plants under LD conditions (Figure 1 and Supplementary Figures 2, 3), suggesting that a faster rate of growth under LD conditions may mask the effect of excessive nitrate on plant growth and flowering. Therefore, the role of nitrate as a nutrient may elucidate the mechanism involved in LD-induced flowering. This notion was further confirmed by the observation that the elevation in ammonium levels, which is an intermediate product of nitrate assimilation, also minimized TLIF (Figure 2B and Supplementary Figure 6). A plausible explanation may be that the role of nitrate as a nutrient source may also act to control the strong nitrate response of SD-dependent flowering.

Besides its role as a nitrogen source, nitrate is also functional in signal-regulated gene expression, metabolism, growth, and development processes in plants (Wang et al., 2012; Bouguyon et al., 2015). Very recently, Olas et al. (2019) observed that flowering was markedly delayed in Arabidopsis $n l p 6 / n l p 7$ double mutants, as there were disruptions in two master regulators of nitrate signaling, demonstrating the vital role of nitrate signaling in regulating the flowering of plants. In this study, we showed that LD-induced flowering may also be associated with the non-nutritional function of nitrate. This was supported by the finding that the sensitivity of the photoperiodic flowering responses to elevated levels of nitrate was reduced by either the replacement of nitrate with ammonium (Figure $\mathbf{2 B}$ and Supplementary Figure 6) or the dysfunction of the nitrate assimilation pathway (Figure 2C and Supplementary Figure 7). In addition, Olas et al. (2019) identified the shoot apical meristem as important site for nitrate signal perception to induce flowering. Our findings that the shoot functions of the NRT1.1 and NRT2.1 were also involved in LD-induced flowering (Figure 6 and Supplementary Figure 11), may also be associated with the role of nitrate in the SAM for floral induction during extended photoperiods. Previous studies have shown that NRT1.1 and NRT2.1 are also expressed in shoots (Guo et al., 2001; Okamoto et al., 2003), indicating that the role of NRT1.1 and NRT2.1 is not limited to nitrate uptake in the roots. As the fundamental functions of both NRT1.1 and NRT2.1 are for nitrate transport across the plasmalemma, it may be a plausible explanation that the NRT1.1 and NRT2.1 in shoots are also involved in distributing nitrate to different shoot tissues. In addition, NRT1.1 is also recognized as a nitrate sensor and NRT2.1 is a putative nitrate sensor (Remans et al., 2006; Ho et al., 2009). The NRT1.1/NRT2.1 in the shoots may also act to transmit nitrate signals to promote flowering. Furthermore, nitrate assimilation may also play a role in the shoot-part function of NRT1.1/NRT2.1 in regulating LD-induced flowering because the distribution of nitrate in different shoot tissues owing to NRT1.1/NRT2.1 could indirectly affect nitrate assimilation. Future studies are required to clarify how the NRT1.1 and NRT2.1 in SAM are involved in LD-induced flowering. 
As previously mentioned, the floral responses to photoperiod alterations and some environmental cues, such as UV light, cold, and salt, are in most cases, under the control of the canonical genetic CO-FT pathway (Guo and Yang, 1998; Martínez et al., 2004; Fornara et al., 2010; Tiwari et al., 2010; Andrés and Coupland, 2012; Luo et al., 2018). FT is a florigen signal that is first expressed in the leaf but induces floral initiation in the SAM (Andrés and Coupland, 2012; Luo et al., 2018). The movement of FT protein through the phloem to the apex has been confirmed in many species (Corbesier et al., 2007; Lin et al., 2007; Aki et al., 2008). CO is another key regulator that encodes a nuclear protein and positively regulates the flowering of plants by increasing FT expression (Putterill et al., 1995). Briefly, light promotes the accumulation of $\mathrm{CO}$. The $\mathrm{CO}$ then interacts with the $\mathrm{B}$ and $\mathrm{C}$ subunits of nuclear factor $\mathrm{Y}$ (NF-Y), to form the NFCO complex, which is bound to the proximal region of the FT promoter and acts to increase FT expression (Tiwari et al., 2010; Andrés and Coupland, 2012; Luo et al., 2018). Very recently, Teng et al. (2019) showed that NRT1.1 regulates flowering in an FTdependent but CO-independent manner. It is worth noting that a disruption in NRT1.1 induced the expression of NRT2.1, and thus the nitrate uptake of the nrt1.1 mutant was significantly improved under nitrate-limited conditions (Muños et al., 2004; Bouguyon et al., 2015; Ye et al., 2019). The induction of NRT2.1 may interfere with the regulation of NRT1.1 for CO expression, under suboptimal nitrate conditions. Therefore, in this study, we observed that the LD-induced expressions of CO and FT were both decreased by the dysfunction of the nitrate transporters NRT1.1 and NRT2.1 (Figure 7). These results indicate that the action of nitrate in LD-induced flowering probably depends on the up-regulation of the CO-FT pathway. This notion was supported by the observation that the flowering that was induced by increasing the nitrate levels was restrained by the loss of function of either CO or FT under LD conditions (Figure 8). The link between nitrate availability and FT expression was also indicated in a previous study by Gras et al. (2018). They found that the mRNA expression of FT peaked when plants were grown in optimal nitrate concentrations, but not at higher concentrations. In addition, they showed that the flowering time of $f t-10$ did not respond to the nitrate supply above the optimal nitrate level. Moreover, nitrate-controlled FT expression was associated with the floral repressors SMZ and SNZ, as the early accumulation of these repressors in high nitrate concentrations repressed FT induction and delayed flowering. However, in this study, we observed that increasing the nitrate concentrations, below the optimal level, still led to earlier flowering in both $\mathrm{co}-1$ and $f t-10$ mutants under LD conditions; although this induction was largely lower than that in the Col-0 plants (Figure 8). These results suggested that the up-regulation of $C O$ and FT by nitrate has a role in promoting flowering under LD conditions.

Although several NRTs have been characterized to be involved in root nitrate uptake in Arabidopsis plants, NRT1.1 and NRT2.1 contribute to the majority of root nitrate uptake under both nitrate-limited and nitrate-sufficient conditions (Li et al., 2006; Wang et al., 2012; Ye et al., 2019). Interestingly, the LD conditions clearly increased the root nitrate uptake by the up-regulation of NRT1.1 and NRT2.1 expression (Figures 3, 4). This led to speculation as to how the LD conditions increased the nitrate uptake using NRT1.1 and NRT2.1. It is widely acknowledged that carbon photo-assimilates are vital signals involved in nitrate uptake, and that extended photoperiod conditions elevate the accumulation of carbon photo-assimilates (Nunes-Nesi et al., 2010; Chen et al., 2016). This suggests that the LD-improved root nitrate uptake may be associated with the increased production of photosynthates in plants. This notion was supported by the finding that the exogenous application of sucrose, a typical photo-assimilate, clearly upregulated the expression of NRT1.1 and NRT2.1, and improved root nitrate uptake in dark-grown Arabidopsis (Lejay et al., 1999, 2003). In contrast, direct regulation of light-responsive transcription factors on nitrate uptake genes may also be an alternative mechanism underlying the improved LD nitrate uptake of the roots. The finding that the light-responsive transcription factor HY5 can directly bind to the promoter of NRT2.1 to improve the expression of NRT2.1, provides evidence to support this speculation (Chen et al., 2016). In addition, three putative binding sites for HY5 have also been identified in the promoter region of NRT1.1, which underpinned the hypothesis that the regulation of HY5 on the expression of NRT1.1, may involve direct binding to the NRT1.1 promoter (Lee et al., 2007; Jonassen et al., 2009).

In summary, improved nitrate status plays a role in LDinduced flowering in Arabidopsis plants. To understand if the nitrate status related to the LD-induced flowering in Arabidopsis plants could be generalized to other plants, multiple species need to be tested in a range of ecosystems. As previously mentioned, plants usually face $\mathrm{N}$ insufficiencies in most ecosystems; however, the global emission and deposition levels of fixed atmospheric nitrogen have been markedly enhanced over the past 70 years because of the development of intensive agriculture and the combustion of fossil fuels (Galloway, 1995; Stevens et al., 2004). If the photoperiodic flowering responses in other plants can also be adjusted by altering the nitrate supply, our results may provide a physiological basis for the effects of $\mathrm{N}$ depositions on plant reproductive dynamics and enable improved predictions of the future changes in the natural reproductive rhythms of plant populations in most terrestrial ecosystems. In addition, our findings may also help to develop more accurate nitrate management protocols to improve crop performance, by controlling the timing of flowering in crops.

\section{DATA AVAILABILITY STATEMENT}

The original contributions presented in the study are included in the article/Supplementary Material, further inquiries can be directed to the corresponding author.

\section{AUTHOR CONTRIBUTIONS}

JY, WT, and CJ executed the experiments, interpreted the data, and generated the figures. MZ assisted in generating nrt1.11/nrt2.1-1 mutants and performing the grafted experiment. QZ and WD assisted in the analysis of data. JY and CJ wrote the 
manuscript. All authors contributed to the article and approved the submitted version.

\section{FUNDING}

This work was financially supported by the Natural Key R\&D Program of China (2016YFD0200103) and the Zhejiang Province Natural Science Foundation (grant no. LZ21D010001).

\section{ACKNOWLEDGMENTS}

We thank Y. R. Hu (Xishuangbanna Tropical Botanical Garden, Chinese Academy of Sciences, China) for kindly providing the $f t-10$ and $c o-1$ lines in a Col-0 background, S. Yuan (Sichuan Agricultural University, China), and B. Liu (Chinese Academy of Agricultural Sciences, Beijing, China) for the cryl line.

\section{SUPPLEMENTARY MATERIAL}

The Supplementary Material for this article can be found online at: https://www.frontiersin.org/articles/10.3389/fpls.2021. 629857/full\#supplementary-material

Supplementary Figure 1 | Flowering time of Arabidopsis Col-0 plants grown under SD (A) and LD (B) conditions.

Supplementary Figure 2 | Effects of nitrate availability on the growth of Arabidopsis Col-0 plants.

\section{REFERENCES}

Achard, P., Cheng, H., De Grauwe, L., Decat, J., Schoutteten, H., Moritz, T., et al. (2006). Integration of plant responses to environmentally activated phyto-hormonal signals. Science 311, 91-94. doi: 10.1126/science.111 8642

Aki, T., Shigyo, M., Nakano, R., Yoneyama, T., and Yanagisawa, S. (2008). Nano scale proteomics revealed the presence of regulatory proteins including three FT-like proteins in phloem and xylem saps from rice. Plant Cell Physiol. 49, 767-790. doi: 10.1093/pcp/pcn049

Alboresi, A., Gestin, C., Leydecker, M. T., Bedu, M., Meyer, C., and Truong, H. N. (2005). Nitrate, a signal relieving seed dormancy in Arabidopsis. Plant Cell Environ. 28, 500-512. doi: 10.1111/j.1365-3040.2005.01292.x

Andrés, F., and Coupland, G. (2012). The genetic basis of flowering responses to seasonal cues. Nat. Rev. Genet. 13, 627-639. doi: 10.1038/nrg 3291

Andrés, F., Kinoshita, A., Kalluri, N., Fernández, V., Falavigna, V. S., Cruz, T. M. D., et al. (2020). The sugar transporter SWEET10 acts downstream of FLOWERING LOCUS T during floral transition of Arabidopsis thaliana. BMC Plant Biol. 20:53. doi: 10.1186/s12870-020-2266-0

Bagnall, D. J. (1993). Light quality and vernalization interact in controlling late flowering in Arabidopsis ecotypes and mutants. Ann. Bot. (Lond). 71, 75-83. doi: 10.1006/anbo.1993.1009

Bao, S., Hua, C., Huang, G., Cheng, P., Gong, X., Shen, L., et al. (2019). Molecular basis of natural variation in photoperiodic flowering responses. Dev. Cell 50, 90-101. doi: 10.1016/j.devcel.2019.05.018

Bao, S. J., Hua, C. M., Shen, L. S., and Yu, H. (2020). New insights into gibberellin signaling in regulating flowering in Arabidopsis. J. Integr. Plant Biol. 62, 118131. doi: 10.1111/jipb.12892

Bouguyon, E., Brun, F., Meynard, D., Kubes, M., Pervent, M., Leran, S., et al. (2015). Multiple mechanisms of nitrate sensing by Arabidopsis nitrate transceptor NRT1.1. Nat. Plants 1:15015. doi: 10.1038/nplants.2015.15
Supplementary Figure 3 | Effects of photoperiod on the bolting time of Arabidopsis Col-0 plants under a wide range of nitrate supplies.

Supplementary Figure 4 | Effects of nitrate availability on the flowering time of Arabidopsis Col-0 plants in the soil system.

Supplementary Figure $\mathbf{5}$ | Effects of nitrate availability on the expression of stress-related marker genes under both SD and LD conditions.

Supplementary Figure 6 | Effects of ammonium availability on the flowering time of Arabidopsis Col-0 plants.

Supplementary Figure 7 | Effects of the dysfunction of nitrate assimilation on the flowering response to nitrate availability.

Supplementary Figure $\mathbf{8}$ | Effects of photoperiod on the expression of nitrate uptake- and assimilation-related genes.

Supplementary Figure 9 | Disruption of NRT1.1 and NRT2.1 functions in nrt1.1-1/2.1-2 mutants in the soil system delayed flowering.

Supplementary Figure 10| The flowering response of $n r t 1.1-1 / 2.1-2$ mutants to nitrate availability in agar system.

Supplementary Figure 11 | Flowering time of grafted plants under $1 \mathrm{mM}$ nitrate conditions.

Supplementary Figure 12 | Expression of flowering-related genes in both Col-0 plants and nrt1.1-1/2.1-2 mutants.

Supplementary Table 1 | Primers used in quantitative real-time polymerase chain reaction.

Supplementary Table 2 | Effects of nitrate availability on the number of rosette leaves of $\mathrm{ft}-10$ and $\mathrm{co}-1$ mutants.

Supplementary Table 3 | Effects of nitrate availability on flowering of the cry1 mutants.

Brady, S. M., Burow, M., Busch, W., Carlborg, Ö, Denby, K. J., Glazebrook, J., et al. (2015). Reassess the $t$ test: interact with all your data via ANOVA. Plant Cell 27, 2088-2094. doi: 10.1105/tpc.15.00238

Capovilla, G., Schmid, M., and Pose, D. (2014). Control of flowering by ambient temperature. J. Exp. Bot. 66, 59-69. doi: 10.1093/jxb/eru416

Castro Marín, I., Loef, I., Bartetzko, L., Searle, I., Coupland, G., Stitt, M., et al. (2011). Nitrate regulates floral induction in Arabidopsis, acting independently of light, gibberellin and autonomous pathways. Planta 233, 539-552. doi: 10 . 1007/s00425-010-1316-5

Chen, X., Yao, Q., Gao, X., Jiang, C., Harberd, N. P., and Fu, X. (2016). Shoot-toroot mobile transcription factor HY5 coordinates plant carbon and nitrogen acquisition. Curr. Biol. 26, 640-646. doi: 10.1016/j.cub.2015.12.066

Corbesier, L., Vincent, C., Jang, S., Fornara, F., Fan, Q., Searle, I., et al. (2007). FT protein movement contributes to long-distance signaling in floral induction of Arabidopsis. Science 316, 1026-1030. doi: 10.1126/science.1140035

Crawford, N. M., and Glass, A. D. M. (1998). Molecular and physiological aspects of nitrate uptake in plants. Trends Plant Sci. 3, 389-395. doi: 10.1016/S13601385(98)01311-9

Delhon, P., Gojon, A., Tillard, P., and Passama, L. (1995). Diurnal regulation of $\mathrm{NO}_{3}{ }^{-}$uptake in soybean plants I. changes in $\mathrm{NO}_{3}{ }^{-}$influx, efflux, and $\mathrm{N}$ utilization in the plant during the day/night cycle. J. Exp. Bot. 46, 1585-1594. doi: $10.1093 /$ jxb/46.10.1585

Desikan, R., Griffiths, R., Hancock, J., and Neill, S. (2002). A new role for an old enzyme: nitrate reductase-mediated nitric oxide generation is required for abscisic acid-induced stomatal closure in Arabidopsis thaliana. Proc. Natl. Acad. Sci. U.S.A. 99, 16314-16318. doi: 10.1073/pnas.252461999

Fang, X. Z., Tian, W. H., Liu, X. X., Lin, X. Y., Jin, C. W., and Zheng, S. J. (2016). Alleviation of proton toxicity by nitrate uptake specifically depends on nitrate transporter 1.1 in Arabidopsis. New Phytol. 211, 149-158. doi: 10.1111/nph. 13892

Fornara, F., de Montaigu, A., and Coupland, G. (2010). Snapshot: control of flowering in Arabidopsis. Cell 141, 550-551. doi: 10.1016/j.cell.2010.04.024 
Fornara, F., Panigrahi, K. C., Gissot, L., Sauerbrunn, N., Rühl, M., Jarillo, J. A., et al. (2009). Arabidopsis DOF transcription factors act redundantly to reduce CONSTANS expression and are essential for a photoperiodic flowering response. Dev. Cell 17, 75-86. doi: 10.1016/j.devcel.2009.06.015

Galloway, J. N. (1995). Acid deposition: perspectives in time and space. Water Air Soil Pollut. 85, 15-24. doi: 10.1007/bf00483685

Gnesutta, N., Kumimoto, R. W., Swain, S., Chiara, M., Siriwardana, C., Horner, D. S., et al. (2017). CONSTANS imparts DNA sequence specificity to the histone fold NF-YB/NF-YC dimer. Plant Cell 29, 1516-1532. doi: 10.1105/tpc.16. 00864

Golembeski, G. S., and Imaizumi, T. (2015). Photoperiodic regulation of florigen function in Arabidopsis thaliana. Arabidopsis Book 13:e0178. doi: 10.1199/tab. 0178

Gómez, L. D., Gilday, A., Feil, R., Lunn, J. E., and Graham, I. A. (2010). AtTPS1-mediated trehalose 6-phosphate synthesis is essential for embryogenic and vegetative growth and responsiveness to $\mathrm{ABA}$ in germinating seeds and stomatal guard cells. Plant J. 64, 1-13. doi: 10.1111/j.1365-313X.2010.04312.x

Gras, D. E., Vidal, E. A., Undurraga, S. F., Riveras, E., Moreno, S., DominguezFigueroa, J., et al. (2018). SMZ/SNZ and gibberellin signaling are required for nitrate-elicited delay of flowering time in Arabidopsis thaliana. J. Exp. Bot. 69, 619-631. doi: 10.1093/jxb/erx423

Guo, F. Q., Wang, R., Chen, M., and Crawford, N. M. (2001). The Arabidopsis dualaffinity nitrate transporter gene AtNRT1.1 (CHL1) is activated and functions in nascent organ development during vegetative and reproductive growth. Plant Cell 13, 1761-1777. doi: 10.11054/tpc.010126

Guo, H. W., and Yang, H. Y. (1998). Regulation of flowering time by Arabidopsis photoreceptors. Science 279, 1360-1363. doi: 10.1126/science.279.5355.1360

Hawkins, B. J., Boukcim, H., and Plassard, C. (2008). A comparison of ammonium, nitrate and proton net fluxes along seedling roots of douglas-fir and lodgepole pine grown and measured with different inorganic nitrogen sources. Plant Cell Environ. 31, 278-287. doi: 10.1111/j.1365-3040.2007.01760.x

Ho, C. H., Lin, S. H., Hu, H. C., and Tsay, Y. F. (2009). CHL1 functions as a nitrate sensor in plants. Cell 138, 1184-1194. doi: 10.1016/j.cell.2009.07.004

Jonassen, E. M., Sévin, D. C., and Lillo, C. (2009). The bZIP transcription factors $\mathrm{HY} 5$ and $\mathrm{HYH}$ are positive regulators of the main nitrate reductase gene in Arabidopsis leaves, NIA2, but negative regulators of the nitrate uptake gene NRT1.1. J. Plant Physiol. 166, 2071-2076. doi: 10.1016/j.jplph.2009.05.010

Kant, S., Peng, M., and Rothstein, S. J. (2011). Genetic regulation by NLA and microRNA827 for maintaining nitrate-dependent phosphate homeostasis in Arabidopsis. PLoS Genet. 7:1002021. doi: 10.1371/journal.pgen.1002021

Kazan, K., and Lyons, R. (2016). The link between flowering time and stress tolerance. J. Exp. Bot. 67, 47-60. doi: 10.1093/jxb/erv441

Kim, J. J., Lee, J. H., Kim, W., Jung, H. S., Huijser, P., and Ahn, J. H. (2012). The microRNA156-SQUAMOSA PROMOTER BINDING PrOTEIN-LIKE3 module regulates ambient temperature-responsive flowering via FLOWERING LOCUS T in Arabidopsis. Plant Physiol. 159, 461-478. doi: 10.1104/pp.111. 192369

Konishi, M., and Yanagisawa, S. (2013). Arabidopsis NIN-like transcription factors have a central role in nitrate signalling. Nat. Commun. 4:1617. doi: 10.1038/ ncomms 2621

Koornneef, M., Hanhart, C. J., and van der Veen, J. H. (1991). A genetic and physiological analysis of late flowering mutants in Arabidopsis thaliana. Mol. Gen. Genet. 229:57. doi: 10.1007/BF00264213

Krapp, A., David, L. C., Chardin, C., Girin, T., Marmagne, A., Leprince, A. S., et al. (2014). Nitrate transport and signalling in Arabidopsis. J. Exp. Bot. 65, 789-798. doi: $10.1093 / \mathrm{jxb} / \mathrm{eru} 001$

Lee, J., He, K., Stolc, V., Lee, H., Figueroa, P., Gao, Y., et al. (2007). Analysis of transcription factor HY5 genomic binding sites revealed its hierarchical role in light regulation of development. Plant Cell 19, 731-749. doi: 10.1105/tpc.106. 047688

Lejay, L., Gansel, X., Cerezo, M., Tillard, P., Muller, C., Krapp, A., et al. (2003). Regulation of root ion transporters by photosynthesis: functional importance and relation with hexokinase. Plant Cell 15, 2218-2232. doi: 10.1105/tpc. 013516

Lejay, L., Tillard, P., Lepetit, M., Olive, F. D., Filleur, S., Daniel Vedele, F., et al. (1999). Molecular and functional regulation of two $\mathrm{NO}_{3}{ }^{-}$uptake systems by N- and C-status of Arabidopsis plants. Plant J. 18, 509-519. doi: 10.1046/j.1365313X.1999.00480.x
Lezhneva, L., Kiba, T., Feria-Bourrellier, A., Lafouge, F., Boutet-Mercey, S., Zoufan, P., et al. (2014). The Arabidopsis nitrate transporter NRT2.5 plays a role in nitrate acquisition and remobilization in nitrogen-starved plants. Plant J. 80, 230-241. doi: 10.1111/tpj.12626

Li, W., Wang, Y., Okamoto, M., Crawford, N. M., Siddiqi, M. Y., and Glass, A. D. M. (2006). Dissection of the AtNRT2.1-AtNRT2.2 inducible high-affinity nitrate transporter gene cluster. Plant Physiol. 143, 425-433. doi: 10.1104/pp. 106.091223

Lin, M. K., Belanger, H., Lee, Y. J., Varkonyi-Gasic, E., Taoka, K. I., Miura, E., et al. (2007). Flowering locus T protein may act as the long-distance florigenic signal in the cucurbits. Plant Cell 19, 1488-1506. doi: 10.1105/tpc.107.051920

Lin, Y. L., and Tsay, Y. F. (2017). Influence of differing nitrate and nitrogen availability on flowering control in Arabidopsis. J. Exp. Bot. 68, 2603-2609. doi: 10.1093/jxb/erx053

Liu, T., Li, Y., Ren, J., Qian, Y., Yang, X., Duan, W., et al. (2013). Nitrate or $\mathrm{NaCl}$ regulates floral induction in Arabidopsis thaliana. Biologia 68, 215-222. doi: 10.2478/s11756-013-0004-x

Luo, X., Gao, Z., Wang, Y., Chen, Z., Zhang, W., Huang, J., et al. (2018). The NUCLEAR FACTOR-CONSTANS complex antagonizes polycomb repression to de-repress FLOWERING LOCUS T expression in response to inductive long days in Arabidopsis. Plant J. 95, 17-29. doi: 10.1111/tpj.13926

Maeda, Y., Konishi, M., Kiba, T., Sakuraba, Y., Sawaki, N., Kurai, T., et al. (2018). A NIGT1-centred transcriptional cascade regulates nitrate signalling and incorporates phosphorus starvation signals in Arabidopsis. Nat. Commun. 9, 1-14. doi: 10.1038/s41467-018-03832-6

Marsch-Martínez, N., Franken, J., Gonzalez-Aguilera, K. L., de Folter, S., Angenent, G., and Alvarez-Buylla, A. E. R. (2013). An efficient flat-surface collar-free grafting method for Arabidopsis thaliana seedlings. Plant Methods 9:14. doi: 10.1186/1746-4811-9-14

Martínez, C., Pons, E., Prats, G., and León, J. (2004). Salicylic acid regulates flowering time and links defence responses and reproductive development. Plant J. 37, 209-217. doi: 10.1046/j.1365-313X.2003.01954.x

Mathieu, J., Yant, L. J., Mürdter, F., Küttner, F., and Schmid, M. (2009). Repression of flowering by the miR172 target SMZ. PLoS Biol. 7:e1000148. doi: 10.1371/ journal.pbio. 1000148

Miller, A. J., and Cramer, M. D. (2005). Root nitrogen acquisition and assimilation. Plant Soil 274, 1-36. doi: 10.1007/s11104-004-0965-1

Moghaddam, M. R. B., and Ende, W. V. D. (2013). Sugars, the clock and transition to flowering. Front. Plant Sci. 4:22. doi: 10.3389/fpls.2013.00022

Muños, S., Cazettes, C., Fizames, C., Gaymard, F., Tillard, P., Lepetit, M., et al. (2004). Transcript profiling in the chl1-5 mutant of Arabidopsis reveals a role of the nitrate transporter NRT1.1 in the regulation of another nitrate transporter, NRT2. 1. Plant Cell 16, 2433-2447. doi: 10.1105/tpc.104.024380

Nunes-Nesi, A., Fernie, A. R., and Stitt, M. (2010). Metabolic and signaling aspects underpinning the regulation of plant carbon nitrogen interactions. Mol. Plant 3, 973-996. doi: 10.1093/mp/ssq049

Ohto, M., Onai, K., Furukawa, Y., Aoki, E., Araki, T., and Nakamura, K. (2001). Effects of sugar on vegetative development and floral transition in Arabidopsis. Plant Physiol. 127, 252-261. doi: 10.1104/pp.127.1.252

Okamoto, M., Vidmar, J. J., and Glass, A. D. (2003). Regulation of NRT1 and NRT2 gene families of Arabidopsis thaliana: responses to nitrate provision. Plant Cell Physiol. 44, 304-317. doi: 10.1093/pcp/pcg036

Olas, J. J., Van Dingenen, J., Abel, C., Działo, M. A., Feil, R., Krapp, A., et al. (2019). Nitrate acts at the Arabidopsis thaliana shoot apical meristem to regulate flowering time. New Phytol. 223, 814-827. doi: 10.1111/nph.15812

Peuke, A. D., and Jeschke, W. D. (1998). The effects of light on induction, time courses, and kinetic patterns of net nitrate uptake in barley. Plant Cell Environ. 21, 765-774. doi: 10.1046/j.1365-3040.1998.00329.x

Pouteau, S., and Albertini, C. (2009). The significance of bolting and floral transitions as indicators of reproductive phase change in Arabidopsis. J. Exp. Bot. 60, 3367-3377. doi: 10.1093/jxb/erp173

Pouteau, S., Ferret, V., Gaudin, V., Lefebvre, D., Sabar, M., Zhao, G. C., et al. (2004). Extensive phenotypic variation in early flowering mutants of Arabidopsis. Plant Physiol. 135, 201-211. doi: 10.1104/pp.104.039453

Putterill, J., Robson, F., Lee, K., Simon, R., and Coupland, G. (1995). The CONSTANS gene of Arabidopsis promotes flowering and encodes a protein showing similarities to zinc finger transcription factors. Cell 80, 847-857. doi: 10.1016/0092-8674(95)90288-0 
Remans, T., Nacry, P., Pervent, M., Filleur, S., Diatloff, E., Mounier, E., et al. (2006). The Arabidopsis NRT1.1 transporter participates in the signaling pathway triggering root colonization of nitrate-rich patches. Proc. Natl. Acad. Sci. U.S.A. 103, 19206-19211. doi: 10.1073/pnas.0605275103

Samach, A., Onouchi, H., Gold, S. E., Ditta, G. S., Schwarz-Sommer, Z., Yanofsky, M. F., et al. (2000). Distinct roles of CONSTANS target genes in reproductive development of Arabidopsis. Science 288, 1613-1616. doi: 10.1126/science.288. 5471.1613

Sawa, M., Nusinow, D. A., Kay, S. A., and Imaizumi, T. (2007). FKF1 and GIGANTEA complex formation is required for day-length measurement in Arabidopsis. Science 318, 261-265. doi: 10.1126/science.1146994

Seligman, K., Saviani, E. E., Oliveira, H. C., Pinto-Maglio, C. A. F., and Salgado, I. (2008). Floral transition and nitric oxide emission during flower development in Arabidopsis thaliana is affected in nitrate reductase-deficient plants. Plant Cell Physiol. 49, 1112-1121. doi: 10.1093/pcp/pcn089

Shabala, L., Zhang, J. Y., Pottosin, I., Bose, J., Zhu, M., Fuglsang, A. T., et al. (2016). Cell-type-specific $\mathrm{H}^{+}$-ATPase activity in root tissues enables $\mathrm{K}^{+}$retention and mediates acclimation of barley (Hordeum vulgare) to salinity stress. Plant Physiol. 172, 2445-2458. doi: 10.1104/pp.16.01347

Srikanth, A., and Schmid, M. (2011). Regulation of flowering time: all roads lead to Rome. Cell Mol. Life Sci. 68, 2013-2037. doi: 10.1007/s00018-011-0673-y

Stevens, C. J., Dise, N. B., Mountford, J. O., and Gowing, D. J. (2004). Impact of nitrogen deposition on the species richness of grasslands. Science 303, 1876-1879. doi: 10.1126/science.1094678

Suárez-López, P., Wheatley, K., Robson, F., Onouchi, H., Valverde, F., and Coupland, G. (2001). CONSTANS mediates between the circadian clock and the control of flowering in Arabidopsis. Nature 410, 1116-1120. doi: 10.1038/ 35074138

Takeno, K. (2016). Stress-induced flowering: the third category of flowering response. J. Exp. Bot. 67, 4925-4934. doi: 10.1093/jxb/erw272

Teng, Y. B., Liang, Y., Wang, M. Y., Mai, H. C., and Ke, L. P. (2019). Nitrate transporter 1.1 is involved in regulated flowering time via transcriptional regulation of FLOWERING LOCUS C in Arabidopsis thaliana. Plant Sci. 284, 30-36. doi: 10.1016/j.plantsci.2019.04.002

Tiwari, S. B., Shen, Y., Chang, H., Hou, Y., Harris, A., Ma, S. F., et al. (2010). The flowering time regulator CONSTANS is recruited to the FLOWERING LOCUS T promoter via a unique cis-element. New Phytol. 186, 57-66. doi: 10.1111/j.1469-8137.2010.03251.x
Wahl, V., Ponnu, J., Schlereth, A., Arrivault, S., Langenecker, T., Franke, A., et al. (2013). Regulation of flowering by trehalose-6-phosphate signaling in Arabidopsis thaliana. Science 339, 704-707. doi: 10.1126/science.1230406

Wang, R. C., Tischner, R., Gutiérrez, R. A., Hoffman, M., Xing, X. J., Chen, M. S., et al. (2004). Genomic analysis of the nitrate response using a nitrate reductasenull mutant of Arabidopsis. Plant Physiol. 136, 2512-2522. doi: 10.1104/pp.104. 044610

Wang, Y. Y., Hsu, P. K., and Tsay, Y. F. (2012). Uptake, allocation and signaling of nitrate. Trends Plant Sci. 17, 458-467. doi: 10.1016/j.tplants.2012. 04.006

Wenkel, S., Turck, F., Singer, K., Gissot, L., Le Gourrierec, J., Samach, A., et al. (2006). CONSTANS and the CCAAT box binding complex share a functionally important domain and interact to regulate flowering of Arabidopsis. Plant Cell 18, 2971-2984. doi: 10.1105/tpc.106.043299

Ye, J. Y., Tian, W. H., and Jin, C. W. (2019). A reevaluation of the contribution of NRT1.1 to nitrate uptake in Arabidopsis under low-nitrate supply. FEBS Lett. 15:593. doi: 10.1002/1873-3468.13473

Yuan, S., Zhang, Z. W., Zheng, C., Zhao, Z. Y., Wang, Y., Feng, L. Y., et al. (2016). Arabidopsis cryptochrome 1 functions in nitrogen regulation of flowering. Proc. Natl. Acad. Sci. U.S.A. 113, 7661-7666. doi: 10.1073/pnas.1602004113

Zeng, J., Sheng, H. J., Liu, Y., Wang, Y., Wang, Y., Kang, H. Y., et al. (2017). High nitrogen supply induces physiological responsiveness to long photoperiod in Barley. Front. Plant Sci. 12:569. doi: 10.3389/fpls.2017.00569

Zhu, J., Fang, X. Z., Dai, Y. J., Zhu, Y. X., Chen, H. S., Lin, X. Y., et al. (2019). Nitrate transporter 1.1 alleviates $\mathrm{Pb}$ toxicity in Arabidopsis by preventing rhizosphere acidification. J. Exp. Bot. 70, 6363-6374. doi: 10.1093/jxb/erz374

Conflict of Interest: The authors declare that the research was conducted in the absence of any commercial or financial relationships that could be construed as a potential conflict of interest.

Copyright (c) $2021 \mathrm{Ye}$, Tian, Zhou, Zhu, Du and Jin. This is an open-access article distributed under the terms of the Creative Commons Attribution License (CC BY). The use, distribution or reproduction in other forums is permitted, provided the original author(s) and the copyright owner(s) are credited and that the original publication in this journal is cited, in accordance with accepted academic practice. No use, distribution or reproduction is permitted which does not comply with these terms. 